\title{
Feedforward Delay Estimators in Adverse Multipath Propagation for Galileo and Modernized GPS Signals
}

\author{
Elena Simona Lohan, Abdelmonaem Lakhzouri, and Markku Renfors \\ Institute of Communications Engineering, Tampere University of Technology, P.O. Box 553, Tampere 33101, Finland
}

Received 31 May 2005; Revised 8 March 2006; Accepted 29 March 2006

\begin{abstract}
The estimation with high accuracy of the line-of-sight delay is a prerequisite for all global navigation satellite systems. The delay locked loops and their enhanced variants are the structures of choice for the commercial GNSS receivers, but their performance in severe multipath scenarios is still rather limited. The new satellite positioning system proposals specify higher code-epoch lengths compared to the traditional GPS signal and the use of a new modulation, the binary offset carrier (BOC) modulation, which triggers new challenges in the delay tracking stage. We propose and analyze here the use of feedforward delay estimation techniques in order to improve the accuracy of the delay estimation in severe multipath scenarios. First, we give an extensive review of feedforward delay estimation techniques for CDMA signals in fading channels, by taking into account the impact of BOC modulation. Second, we extend the techniques previously proposed by the authors in the context of wideband CDMA delay estimation (e.g., Teager-Kaiser and the projection onto convex sets) to the BOC-modulated signals. These techniques are presented as possible alternatives to the feedback tracking loops. A particular attention is on the scenarios with closely spaced paths. We also discuss how these feedforward techniques can be implemented via DSPs.
\end{abstract}

Copyright (c) 2006 Elena Simona Lohan et al. This is an open access article distributed under the Creative Commons Attribution License, which permits unrestricted use, distribution, and reproduction in any medium, provided the original work is properly cited.

\section{BACKGROUND AND MOTIVATION}

Applications of GNSS are rapidly evolving. A new European satellite system, Galileo, is currently in standardization process $[1,2]$. Modernized GPS proposals have also been introduced recently [3-5]. Galileo signals, as well as GPS signals, are based on direct-sequence code division multiple access (DS-CDMA) technique. Spread spectrum systems are known to offer better frequency reuse, better multipath diversity, better narrowband interference rejection, and, potentially, better capacity compared to narrowband techniques [6]. On the other hand, code and frequency synchronization are fundamental prerequisites for a good performance of the receiver. These two tasks pose several problems in the presence of mobile wireless channels, due to the various adverse effects of the channel, such as the multipath propagation, the possibility of having the line-of-sight (LOS) component obstructed by closely spaced non-line-of-sight (NLOS) components, or even the absence of LOS, and the high level of noise (especially in indoor scenarios). Moreover, the fading statistics of the channel and the possible variations of the oscillator clock limit the coherent integration length at the receiver (i.e., the receiver filters which are used to smooth the various estimates of channel parameters cannot have the bandwidth smaller than the maximum Doppler spread of the channel without introducing significant errors in the estimation process) [7-11]. The Doppler shift induced by the satellite movement is also prone to deteriorate the receiver performance, unless correctly estimated and removed. Moreover, the fading behavior of the channel paths induces a certain Doppler spread, directly related to the terminal velocity. Typical GNSS receivers estimate jointly the code phase and the Doppler shifts/spreads via a two-dimensional search in time-frequency plane. The delay-Doppler estimation is usually done in two stages: acquisition (or coarse estimation), followed by tracking (or fine estimation). The acquisition and tracking stages will be treated here together, assuming implicitly that the frequency-time search space is reduced, for example, via some assistance data (e.g., Doppler assistance, knowledge of previous delay estimates, etc.). In this situation, the delay estimation problem can be seen as a tracking problem (i.e., very accurate delay estimates are desired) with initial code misalignment of several chips or tens of chips and initial Doppler shift not higher than few tens of Hertz.

One particular situation in multipath propagation is the situation when LOS component is overlapping with one or several closely spaced NLOS components [7,9-16], making the delay estimation process more difficult. This closely 
spaced path scenario is likely to be encountered in indoor positioning applications or in outdoor urban environments, and will be the main focus of our paper.

The multipath delay estimation problem (including closely spaced path situation) has been widely studied for terrestrial CDMA receivers (e.g., WCDMA) and for the traditional C/A GPS signal. Nevertheless, the introduction of the new modulation type, namely, the BOC modulation (both sine and cosine $\mathrm{BOC}$ variants) has triggered new potential challenges in the delay-Doppler estimation process. BOC modulation has been proposed in [4] in order to improve the spectral efficiency of the L band, by moving the signal energy away from the band center, thus offering a higher degree of spectral separation between BOC-modulated signals and the other signals which use traditional phase-shift-keying modulation. Recently, BOC modulation has been selected in most of the proposals regarding Galileo and modernized GPS signals $[1,2,5]$.

The main algorithms used for GPS and Galileo code tracking, provided a certain sufficiently small Doppler shift, are based on what is typically called a feedback delay estimator and they are implemented based on a feedback loop. The most known feedback delay estimators are the delay-locked loops (DLLs) [13, 17-21]. The classical DLLs fail to cope with multipath propagation [6]. Therefore, several enhanced DLL-based techniques have been introduced in order to mitigate the effect of multipaths, especially in closely spaced path scenarios.

One class of these enhanced DLL techniques is based on the idea of narrowing the spacing between early and late correlators (i.e., narrow correlator class) [22-24]. Another class of enhanced DLL structures uses a modified reference waveform for the correlation at the receiver, that narrows the main lobe of the cross-correlation function, at the expense of a deterioration of signal power. Examples belonging to this class are the gated correlator [24], the strobe correlators $[23,25]$, the pulse aperture correlator [26], and the modified correlator reference waveform [23, 27]. Another category of improved DLL techniques uses some form of multipath interference cancellation, by estimating not only the delay of the LOS path, but also the delays, phases, and amplitudes of the NLOS paths $[13,21,28]$.

Another family of the feedback delay estimators is based on the extended Kalman filters (EKF) and it has been studied in the context of WCDMA systems $[8,9,29,30]$. The EKF approach was shown to provide accurate delay estimates in the presence of closely spaced paths and to converge fast to the correct solution. However, due to the complexity and to the high sensitivity of the EKF algorithm to the initialization conditions, such as the error covariance matrices [8], the use of EKF estimators is not widespread in the today's research community. Moreover, since their complexity is directly related to the code epoch length (or, equivalently, the spreading factor), EKF estimators are clearly not suitable for Galileo and modernized GPS applications.

An alternative to the above-mentioned feedback loop solutions is based on the open-loop (or feedforward) solutions, which constitutes the topic of our study. Feedforward solu- tions refer to the solutions which make the delay estimation in a single step, without requiring a feedback loop. A general classification of open-loop solutions for WCDMA applications can be found in $[9,30]$. Among the open-loop solutions, we mention the deconvolution algorithms, the Teager-Kaiser (TK)-based algorithms, the subspace-based approaches, the algorithms based on quadratic programming (QP), and the suboptimal ML-based algorithms [9, 3032]. The subspace-based solutions seem infeasible for GNSS applications nowadays, due to their high complexity (proportional to the length of the code epoch in samples). The QP and ML-based solutions were shown in $[9,30]$ to give worse results than TK and POCS algorithms for WCDMA signals.

The most promising approaches in WCDMA applications were found to be the deconvolution algorithms $[7,10]$, and, especially, the projection onto convex sets POCS algorithm $[9,12,14,30,33]$, as well as the Teager-Kaiser-based algorithms $[9,30,34,35]$. These last two approaches (POCS and TK) proved to give the best results for WCDMA scenarios in the presence of overlapping paths $[9,30]$.

The feedforward approaches have not been studied yet for BOC-modulated signals. Our paper addresses the problem of estimating the delay of the first arriving path via feedforward approaches, which represent an alternative to the existing feedback solutions. After presenting the signal model in the presence of BOC modulation, we continue with a discussion regarding the advantages and drawbacks of feedback delay estimation algorithms in multipath propagation and we show that feedforward delay estimators may be used as viable alternatives, in order to attain good accuracy via simple implementation. A performance comparison between the feedback and feedforward solutions is out of the scope of this paper, since the assumptions for the two types of methods are clearly different, as it will be explained in Section 3. The main target is to show here the viability of feedforward solutions as delay estimation blocks in modernized GNSS receivers.

We explain how the existing feedforward estimators may be extended in the presence of BOC-modulated pseudorandom (PRN) codes, and we compare their algorithmic and computational performance. We include simulation results showing the performance of various feedforward algorithms in multipath fading channels, as well as the implementational complexity of the most promising feedforward techniques for Galileo and modernized GPS signals, focusing on the programmable type of implementation. The signal used in the simulations and in the complexity calculations is a sine $\operatorname{BOC}(1,1)$-modulated signal, as that one proposed for Galileo open services [2].

In Section 2 we present the signal model in the presence of BOC modulation. Section 3 starts with a discussion regarding the main feedback algorithms (their main advantages and drawbacks), and continues with the comprehensive description of feedforward algorithms that can be used for accurate multipath delay estimation. The description of the cost functions for various feedforward algorithms is given in Section 3.2. Section 3.3 discusses the choice of the threshold needed for feedforward delay estimators: the feedforward 
algorithms are based on the idea that all the local maxima of a certain cost function that are above a threshold are signalling the multipath components. Section 4 compares the feedforward algorithms in terms of detection probability and root-mean-square error and discusses the possible advantages of feedforward delay estimators. Section 5 compares the most promising delay estimation algorithms in terms of execution time and memory requirements, by focusing on the programmable type of implementation, via two fixed point digital signal processors (DSPs) from Texas Instruments: the TMS320C64x and TMS 320C55x families. Section 6 presents the conclusions and the steps to be taken when designing a feedforward delay estimator for positioning applications.

\section{SIGNAL MODEL IN THE PRESENCE OF BOC MODULATION}

For clarity of the notations, the continuous-time model is mostly employed in what follows. The extension to the discrete-time model is straightforward and all the estimation results of this paper are based on the discrete-time implementation.

For simplicity reasons (and due to the fact that Sin$\operatorname{BOC}(1,1)$ modulation is the modulation of choice for Galileo open services), we present here only the case of sine BOC modulation. The extension to cosine BOC modulation is however straightforward, by using the definition of cosine BOC modulation given in $[36,37]$. The sine BOC modulation is a square subcarrier modulation, where the PRN signal (including data modulation) $s_{\mathrm{PRN}}(t)$ is multiplied by a rectangular subcarrier $s_{\mathrm{BOC}}(t)$ of frequency $f_{\mathrm{sc}}$, which splits the spectrum of the signal $[4,5]$. Formally, the sine BOCmodulated PRN waveform $x_{\mathrm{BOC}}(t)$, can be written as the convolution between a PRN sequence $s_{\mathrm{PRN}}(t)$ and a BOC waveform $s_{\mathrm{BOC}}(t)$ as follows $[36,37]$ :

$$
x_{\mathrm{BOC}}(t)=s_{\mathrm{BOC}}(t) \circledast s_{\mathrm{PRN}}(t),
$$

where $[36,37]$

$$
s_{\mathrm{BOC}}(t) \triangleq \sum_{i=0}^{N_{\mathrm{BOC}}-1}(-1)^{i} p_{\mathrm{BOC}}\left(t-i \frac{T_{c}}{N_{\mathrm{BOC}}}\right)
$$

and $\circledast$ is the convolution operator. Above, $T_{c}$ is the chip period and $N_{\mathrm{BOC}}$ is the BOC modulation order, defined as twice the ratio between the subcarrier frequency $f_{\mathrm{sc}}$ and the chip rate $f_{c}$ [4] (i.e., $N_{\mathrm{BOC}}=2 f_{\mathrm{sc}} / f_{c}$ and $N_{\mathrm{BOC}}$ is an integer number). The usual notation for BOC modulation is $\operatorname{BOC}\left(f_{\mathrm{sc}}, f_{c}\right)$. For Galileo signals, the notation $\operatorname{BOC}\left(n_{1}, n_{2}\right)$ is also used, where $n_{1}$ and $n_{2}$ are two indices (not necessarily integers), satisfying the relationships $n_{1}=f_{\mathrm{sc}} / f_{\text {ref }}$ and $n_{2}=f_{c} / f_{\text {ref }}$, respectively, where $f_{\text {ref }}$ is a reference frequency (typically, $\left.f_{\text {ref }}=1.023 \mathrm{MHz}\right)[1,4]$. In $(2), p_{\mathrm{BOC}}(t)$ is a rectangular pulse of support $T_{c} / N_{\text {BOC }}$, namely

$$
p_{\mathrm{BOC}}(t)= \begin{cases}1 & \text { if } 0 \leq t<\frac{T_{c}}{N_{\mathrm{BOC}}} \\ 0 & \text { otherwise. }\end{cases}
$$

Above, $s_{\mathrm{PRN}}(t)$ is the pseudorandom (PRN) code sequence (including the data modulation) of the satellite of interest. The interference of the other satellites is modeled as additive white Gaussian noise here. The data-modulated PRN signal can be written as

$$
\begin{aligned}
s_{\mathrm{PRN}}(t)= & \sum_{n=-\infty}^{+\infty} \sum_{k=1}^{S_{F}} b_{n} c_{k, n} \delta\left(t-n T-k T_{c}\right) \\
& \text { if } N_{\mathrm{BOC}}=1 \text { or } N_{\mathrm{BOC}} \text { even , } \\
s_{\mathrm{PRN}}(t)= & \sum_{n=-\infty}^{+\infty} \sum_{k=1}^{S_{F}} b_{n}(-1)^{n} c_{k, n} \delta\left(t-n T-k T_{c}\right) \\
& \text { if } N_{\mathrm{BOC}} \text { odd and } N_{\mathrm{BOC}}>1,
\end{aligned}
$$

where $b_{n}$ is the data symbol corresponding to the $n$th code epoch (e.g., it is either 1, if no data modulation is present, or constant over $20 \mathrm{~ms}$, if a data rate of $50 \mathrm{bps}$ is employed), $c_{k, n}$ is the $k$ th chip of the $n$th code epoch, $T_{c}$ is the chip interval, $T$ is the code epoch period, $S_{F}$ is the spreading factor or the number of chips per code epochs (i.e., $T=S_{F} T_{c}$ ), and $\delta(\cdot)$ is the Dirac pulse. We remark that an additional factor $(-1)^{n}$ is multiplied with the chip sequence in the lower part of (4), in order to take explicitly into account the odd BOC modulation orders, similar with $[4,38]$. This means that in order to be able to model the BOC modulation in a unified format (for both even and odd BOC modulations, via (1) to (4)), we need the above convention: for odd BOC-modulation orders, the chip sequence is first multiplied with an alternate sequence of $+1 \mathrm{~s}$ and $-1 \mathrm{~s}$ and for even BOC-modulation order, the chip sequence remains unchanged. This multiplication will not change the signal auto- and cross-correlation functions in a significant way, since the randomness of the code is still preserved after chip inversion of every second bit. Also, the power spectral densities will remain unchanged.

An example of sine BOC-modulated waveforms for $N_{\mathrm{BOC}}$ $=1,2,3$ is shown in Figure 1. We remark, from (1), (2), and (4), that $N_{\mathrm{BOC}}=1$ corresponds to a BPSK-modulated PRN sequence.

The normalized baseband power spectral density (PSD) ${ }^{1}$ of a sine BOC-modulated signal is given in $[4,36,37]$ :

$$
\begin{aligned}
& X_{\mathrm{BOC}}(f) \\
& = \begin{cases}\frac{1}{T_{c}}\left(\frac{\sin \left(\pi f T_{c} / N_{\mathrm{BOC}}\right) \sin \left(\pi f T_{c}\right)}{\pi f \cos \left(\pi f T_{c} / N_{\mathrm{BOC}}\right)}\right)^{2}, & N_{\mathrm{BOC}} \text { even, } \\
\frac{1}{T_{c}}\left(\frac{\sin \left(\pi f T_{c} / N_{\mathrm{BOC}}\right) \cos \left(\pi f T_{c}\right)}{\pi f \cos \left(\pi f T_{c} / N_{\mathrm{BOC}}\right)}\right)^{2}, & N_{\mathrm{BOC}} \text { odd } .\end{cases}
\end{aligned}
$$

An example of the PSD for several BOC-modulated signals (with $N_{\mathrm{BOC}}$ from 1 to 4 ) is shown in Figure 2. The situation with $N_{\text {BOC }}=1$ coincides with BPSK modulation (e.g., such as for GPS C/A code). The even-modulation orders ensure a splitting of the spectrum into two symmetrical parts, by moving the energy of the signal away from the DC frequency, and therefore allowing for less interference in the

\footnotetext{
1 The normalization was done with respect to the chip interval $T_{c}$, or, equivalently, to the signal power over infinite bandwidth, similar to [4].
} 


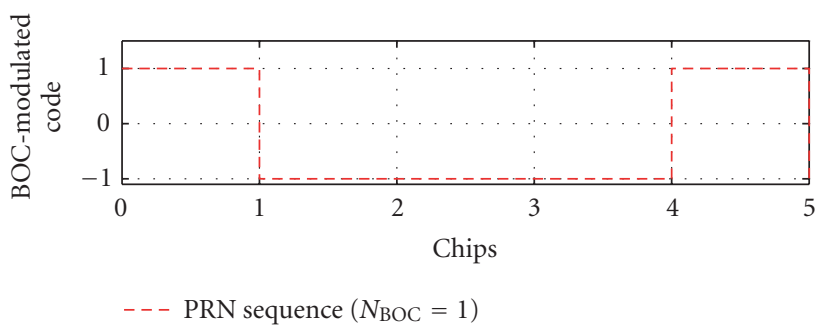

(a)

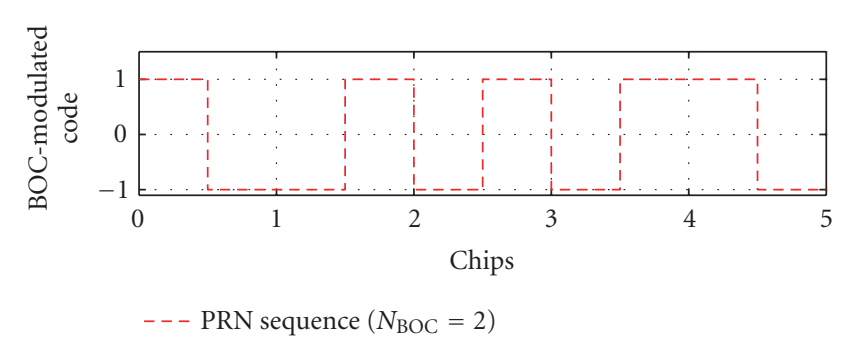

(b)

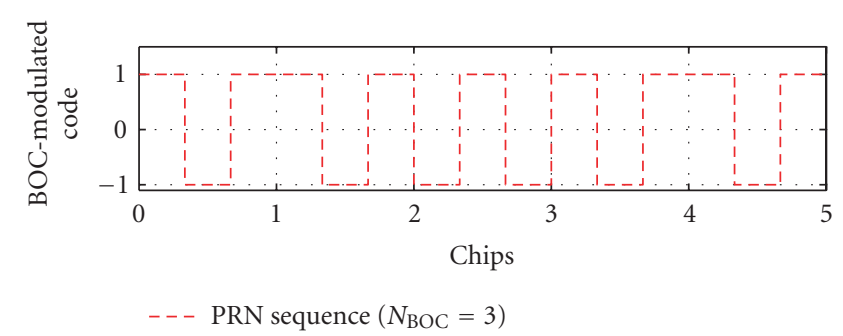

(c)

FIGURE 1: Examples of time-domain waveforms for BOC-modulated signals.

existing GPS bands. The most representative case is that one for $N_{\mathrm{BOC}}=2$, which corresponds to the currently selected modulation format by the Galileo Signal Task Force (i.e., sine $\operatorname{BOC}(1,1))$. The cases with odd modulation index (e.g., $N_{\mathrm{BOC}}=3$ ) do not suppress completely the interference around the DC frequency.

The baseband model of the received signal after the fading channel can be written as

$$
r(t)=\sqrt{E_{b}} e^{+j 2 \pi f_{D} t} \sum_{l=1}^{L} \alpha_{n, l}(t) x_{\mathrm{BOC}}\left(t-\tau_{l}\right)+\eta(t),
$$

where $E_{b}$ is the bit or symbol energy of the signal (one symbol here is equivalent with one code epoch, and it typically has a duration of $T=1 \mathrm{~ms}$ ), $f_{D}$ is the Doppler shift introduced by the channel, $L$ is the number of channel paths, $\alpha_{l, n}(t)$ is the time-varying complex fading coefficient of the $l$ th path during the $n$th code epoch, $\tau_{l}$ is the corresponding path delay (assumed to be constant during the observation inter$\mathrm{val}$ ), and $\eta(\cdot)$ is an additive noise component of double-sided wideband power spectral density $N_{w}$, which incorporates the additive white noise of the channel and the interference coming from the other satellites. We remark that the relationship between the bit energy-to-noise ratio $E_{b} / N_{w}$ (in $\mathrm{dB}$ ) and the

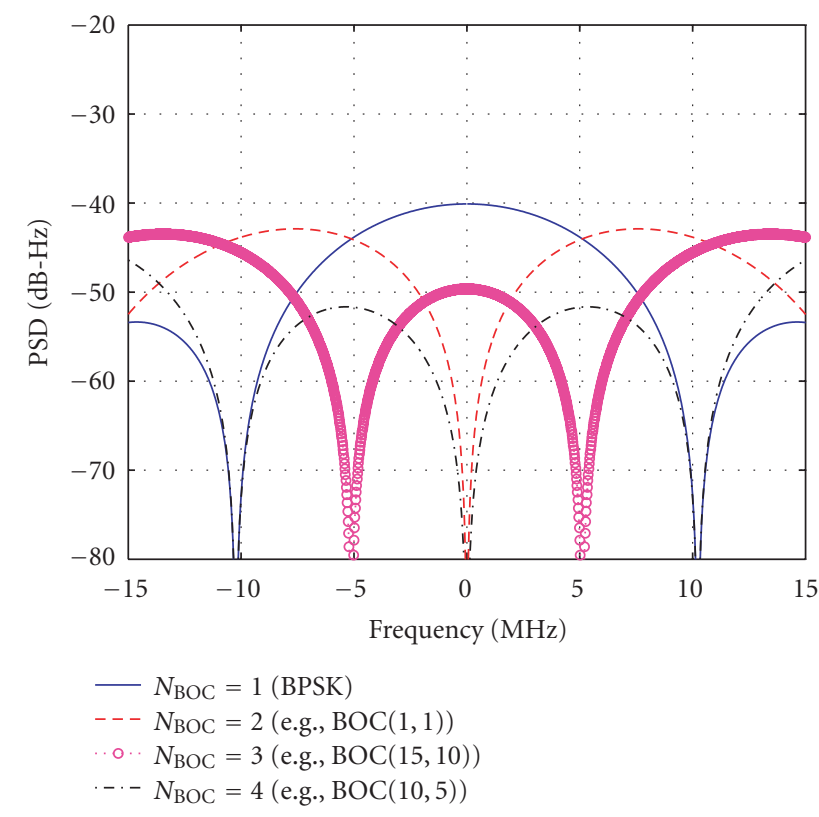

FIGURE 2: Examples of baseband PSD for BOC-modulated signals, $f_{c}=10.23 \mathrm{MHz}$.

carrier-to-noise ratio ( $\mathrm{CNR}$, in $\mathrm{dB}-\mathrm{Hz})$ is [39]

$$
\frac{E_{b}}{N_{w}}[\mathrm{~dB}]=\mathrm{CNR}[\mathrm{dB}-\mathrm{Hz}]+10 \log _{10}\left(T_{c}\right) .
$$

The acquisition and tracking of the received signal are based on the correlation with the reference PRN code with different time lags $\tau$ and frequency shifts $f$. After the data modulation removal, ${ }^{2}$ the correlation with the reference PRN code, and the coherent integration over $N_{c} T$ seconds at the receiver $\left(N_{c}\right.$ is the coherent integration time in code epochs or in $\mathrm{ms}$ if $T=1 \mathrm{~ms}$ ), we can obtain, after straightforward computations, a two-dimensional time-frequency matrix $\mathbf{R}$ with elements $R(f, \tau)$ as follows:

$$
\begin{aligned}
R(f, \tau)= & \sqrt{E_{b}} e^{j \pi\left(f_{D}-f\right) N_{c} T} \operatorname{sinc}\left(\pi\left(f_{D}-f\right) N_{c} T\right) \\
& \times \sum_{l=1}^{L} \tilde{\alpha}_{l} \mathcal{R}_{\mathrm{BOC}}\left(\tau-\tau_{l}\right)+\tilde{\eta}(f, t),
\end{aligned}
$$

where $\operatorname{sinc}(x) \triangleq \sin (x) / x$ and the subscript $n$ has been dropped for simplicity. Above, the filtered noise $\tilde{\eta}(\cdot)$ incorporates the intersymbol interference as well. By virtue of central limit theorem, we assume that $\tilde{\eta}(\cdot)$ is a zero-mean Gaussian noise process. The notation $\widetilde{\alpha}_{l}$ stands for the averaged channel coefficients over $N_{c}$ code epochs. Clearly, if the coherent integration time is higher than the coherence time of the channel, the received signal will be severely distorted. The

\footnotetext{
${ }^{2}$ Here, we assume either that the data bits have been previously estimated and removed from the received signal, or that a pilot signal is available. Errors in data bit estimates are not analyzed here, but may deteriorate the performance of the algorithms.
} 


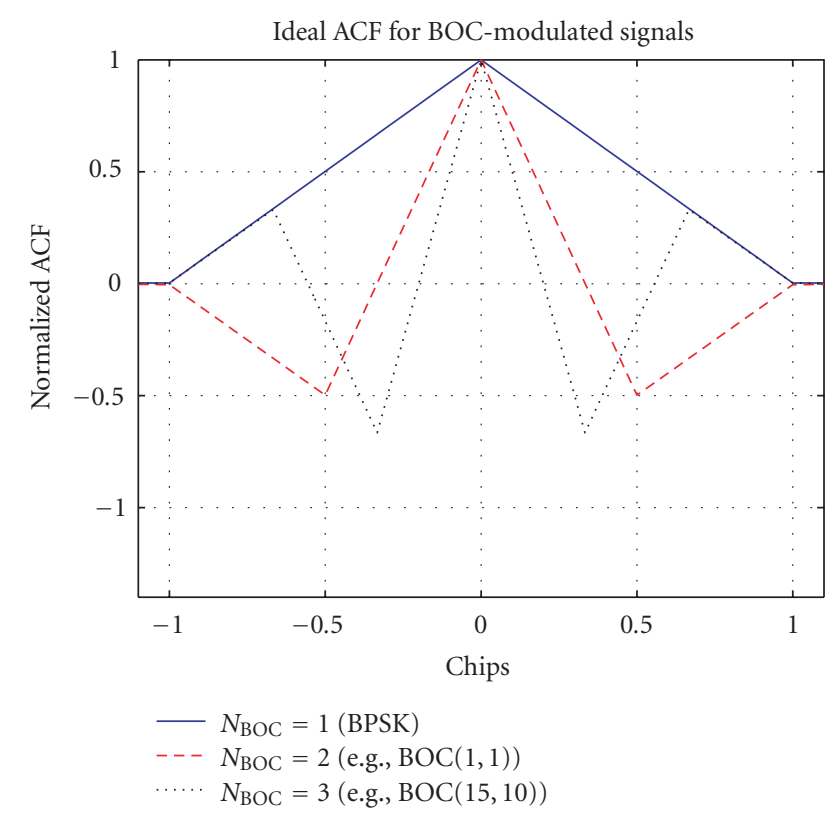

FIgURE 3: Examples of the real part of the ACF for BOC-modulated signals.

term $\operatorname{sinc}\left(\pi\left(f_{D}-f\right) N_{c} T\right)$ in (8) is modeling the deterioration due to a frequency error $f_{D}-f$. In $(8) \mathcal{R}_{\mathrm{BOC}}(\cdot)$ is the ideal ACF of a sine BOC-modulated PRN sequence, given by (direct consequence of (1) and (2), after several manipulations)

$$
\mathcal{R}_{\mathrm{BOC}}(\tau)=\sum_{i=0}^{N_{\mathrm{BOC}}-1} \sum_{j=0}^{N_{\mathrm{BOC}}-1}(-1)^{i+j} \Lambda_{\mathrm{BOC}}\left(\tau-(i-j) T_{\mathrm{BOC}}\right),
$$

and $\Lambda_{\mathrm{BOC}}(\cdot)$ is the triangular-shaped ACF of an ideal PRN sequence of period $T_{\mathrm{BOC}}=T_{c} / N_{\mathrm{BOC}}$ :

$$
\Lambda(\tau)= \begin{cases}\frac{1-|\tau|}{T_{\mathrm{BOC}}} & \text { if }|\tau| \leq T_{\mathrm{BOC}} \\ 0 & \text { otherwise. }\end{cases}
$$

Some examples of the real part of the ideal ACF of BOCmodulated PRN sequences are shown in Figure 3.

The two-dimensional matrix $\mathbf{R}$ with elements given in (8) can be further noncoherently averaged over $N_{n c}$ blocks (i.e., the total coherent and noncoherent integration time will be $N_{c} N_{n c} T$ seconds). The noncoherent averaging may be needed for further noise reduction, because the coherent averaging interval is limited by the coherence time of the fading channel, by the stability of the local oscillator and by the possible residual Doppler shift errors. However, there are some squaring losses in the signal power due to noncoherent averaging. Examples of coherence times $(\Delta t)_{\text {coh }}$ of Galileo channels for a carrier frequency of $f_{\text {carrier }}=1.575 \mathrm{GHz}$ (corresponding to E2-L1-E1 band [2]) are given in Table 1, according to the definition in [40], namely, $(\Delta t)_{\mathrm{coh}} \approx c / v f_{\text {carrier }}$, where $v$ is the ground receiver speed and $c$ is the speed of light. We remark that the coherent integration time should be less than the values given in Table 1, in order to keep the fading spectrum
TABLE 1: Channel coherence times for various receiver speeds for Galileo E2-L1-E1 signal.

\begin{tabular}{ccccccc}
\hline $\begin{array}{c}\text { Speed } \\
(\mathrm{km} / \mathrm{h})\end{array}$ & 2 & 4 & 20 & 40 & 80 & 120 \\
\hline $\begin{array}{c}\text { Coherence } \\
\text { time (ms) }\end{array}$ & 342.8 & 171.42 & 34.28 & 17.14 & 8.57 & 5.71 \\
\hline
\end{tabular}

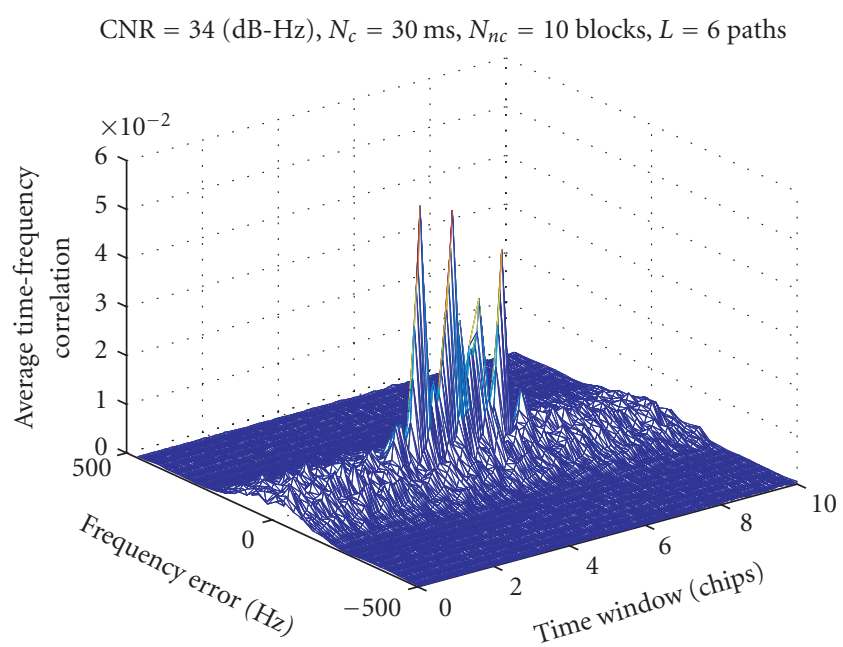

FIGURE 4: Examples of the time-frequency correlation (or matched filter) mesh after coherent and non-coherent integration, 6 closely spaced paths.

of the signal undistorted. Table 1 takes into account only the receiver ground speed. We remark that there is also a relative speed of the mobile receiver with respect to the satellite speed, which is much higher than the receiver ground speed. This will create a Doppler shift effect on the signal (as seen in (6)). Thus, we have both a Doppler shift (due to the satellite movement) and a Doppler spread around the Doppler shift frequency (due to the receiver movement). The Doppler shift should be estimated and removed before the coherent integration (we assume that this has been done in the acquisition stage). If there remains some residual Doppler errors, then the values given in Table 1 become very loose upper bounds on the coherent integration times.

The delay estimation is done on a time-frequency grid whose values are the averaged correlation functions with different time and frequency lags. As seen in (8), the maxima occur at $f=f_{D}$ and $\tau=\tau_{l}$. An example of a time-frequency grid for a 6-path Rayleigh fading channel, covering a frequency offset of $1 \mathrm{kHz}$ and a time window of 10 chips, is shown in Figure 4.

\section{DELAY ESTIMATION ALGORITHMS}

\subsection{Feedback estimators}

Traditionally, the multipath delay estimation block is implemented via a feedback loop. The most common feedback 


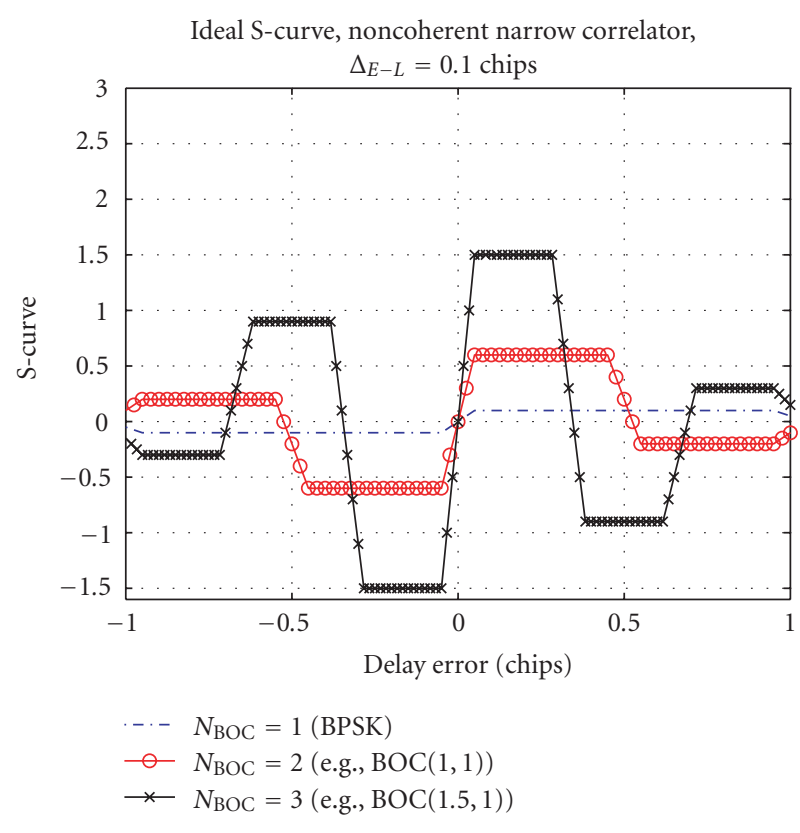

FIGURE 5: Ideal S-curve for BPSK and sine BOC modulations, $\Delta_{E-L}=0.1$ chips.

structures for the delay estimation are the so-called DLLs $[3,5,13,17,20]$. Several enhanced DLLs have been proposed in the presence of multipaths. One example is the narrow correlator [22-24], where the spacing $\Delta_{E-L}$ between early and late correlators is reduced below 1 chip. The performance of narrow correlator is somehow limited in closelyspaced multipath scenarios [23]. Another example is the Rake DLL (RDLL) [21, 28] which uses a separate multipath channel estimation unit which provides the estimates of the interfering path parameters. The estimated parameters are used in a Rake-like structure to resolve and combine the received multipath components. The RDLL is conceptually close to the DLL with interference-cancellation (IC) $[13,17]$. The DLL with IC subtracts the estimated contribution of interfering paths from the output of the finger tracking the path of interest. Another improved variant of DLL is the so-called DLL with interference-minimization (IM) technique [13]. The idea of the DLL with IM is to filter the outputs of the correlators with some adaptive filter, whose coefficients are designed in such a way to minimize the multipath interference. Similar ideas can be found also in the Phase Multipath Mitigation Window Correlator (PMMWC), proposed in [41]. Again, the knowledge about the interfering path parameters should be obtained via an additional multipath channel estimation unit. Since RDLLs, PMMWCs, DLLs with IC and DLLs with IM are conceptually close, we illustrate here the performance of a DLL with IC in the presence of multipaths and BOC modulation.

The performance of the DLL is best illustrated by the socalled S-curve, which presents the expected value of the error signal as a function of the reference parameter error (i.e., the code phase error) [6]. Figure 5 shows the S-curve in singlepath channel for BPSK and two BOC-modulated signals. The

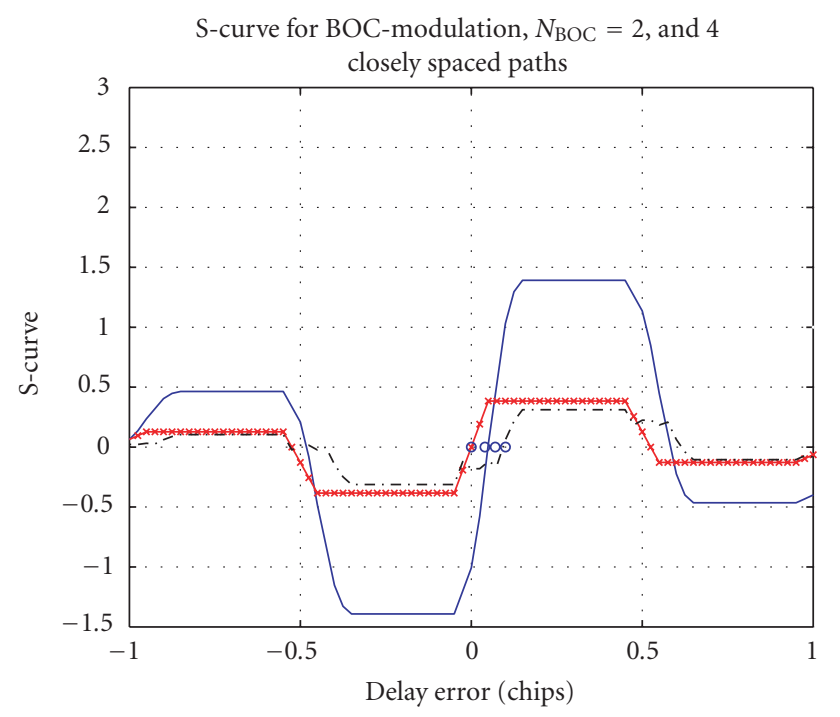

\footnotetext{
- True path delays (with respect to LOS)

- Global S-curve, no interference cancellation (IC)

* S-curve of first path with IC, no channel estimation errors

- - - S-curve of first path with IC and small channel estimation errors (i.e., 0.05 delay error and 0.01 amplitude error)
}

FIgure 6: Performance of a DLL with IC in the presence of multipath channels and BOC modulation $\left(N_{\mathrm{BOC}}=2\right), \Delta_{E-L}=0.1$ chips, channel path delays at $[0,0.04,0.07,0.1] T_{c}$, channel path amplitudes $[0.8,1,0.7,0.4]$.

number of side-lobes increases as the BOC modulation order $N_{\text {BOC }}$ increases. The zero-crossings from below here indicate the presence of a multipath. However, for BOC-modulated signals, the search range should be decreased to less than 2 chips (as it is the case for BPSK modulation). For example, as seen in Figure 5, for $N_{\mathrm{BOC}}=2$ (e.g., $\left.\operatorname{BOC}(1,1)\right)$, the search range should be between $-1 /\left(2 N_{\mathrm{BOC}}\right)$ and $+1 /\left(2 N_{\mathrm{BOC}}\right)$ chips, in order to have convergence and to avoid the false lock points. In order to cope with the side-lobes of the ACF function, a very early-very late (VE-VL) loop with a narrower correlator spacing was proposed for Galileo and modernized GPS signals [3]. The typical DLLs have early, late, and prompt correlators to track the delays. The VE-VL loops introduce two extra correlators (one very early, another one very late) in order to check better that the prompt reference signal is aligned with the main peak of the correlation function, and not a secondary peak. Conceptually, a very earlyvery late DLL is close to the sample-correlate-choose largest (SCCL) algorithm [19] and, to some extent, also to the high resolution correlator (HRC) [24]. However, in VE-VL case, the additional correlators are used only to check that the main peak is on the prompt, but they are not used directly in the tracking [3], while in HRC case, an S-curve is formed based on the 4 correlators (early, late, very-early, and verylate) and the delay is tracked according to this S-curve [24].

If multipath components are present, the performance of an enhanced DLL is shown in Figure 6 (here, a coherent DLL with IC is selected for illustration purpose). The channel has 
4 in-phase static paths, and the first path is weaker than the second one (see Figure 6 caption). In the absence of any IC, the channel paths are merging (here, we showed the situation of closely spaced paths) and the S-curve is not able to track correctly the LOS delay. In the presence of IC, if the multipath channel estimation unit operates perfectly (i.e., no channel estimation errors), the DLL with IC is able to track correctly the LOS component (see Figure 6). However, even small channel estimation errors will destroy completely the ability of the DLL to track the LOS correctly, as shown in Figure 6. For example, the delay error for the narrow correlator (no IC) was 0.05 chips (i.e., $14.66 \mathrm{~m}$ ), and, for DLL with IC and channel estimation errors, it becomes 0.09 chips (i.e., $26.39 \mathrm{~m})$.

To summarize the discussion about feedback tracking loops (i.e., DLLs and their enhanced variants), the main drawbacks of the DLL-based techniques include their reduced ability to deal with closely spaced path scenarios under realistic assumptions (such as the presence of errors in the channel estimation process), their relatively slow convergence, the small pull-in range if small spacing (such as for narrow correlator) is used, and the possibility to lose the lock (i.e., start to estimate the delays with high estimation error) due to the feedback error propagation. Moreover, the DLLbased techniques work only under the assumption that the initial delay error is sufficiently small (e.g., for BOC signals smaller, in absolute value, than $1 /\left(2 N_{\mathrm{BOC}}\right)$ chips due to the fades in the ACF, as seen in Figure 3 ).

Despite their disadvantages, the feedback DLL-based approaches are still the tracking structures of choice for nowadays receivers, due to a number of positive features. Among the advantages of DLLs we have the fact that only 3 correlators are typically needed (or at most 5, e.g., for HRC or VE-VL structures), DLLs behaves good in friendly environments (e.g., distant paths, single path channels, etc.), and there is no need of thresholding as in the case of feedforward techniques (this will be explained in detail in Section 3.3).

It is the purpose of our paper to show that feedforward delay estimation techniques may be, however, feasible alternatives to feedback tracking loops, in terms of good accuracy of the delay estimation process and reasonable complexity, as it will be shown in what follows. Due to the fact that feedback tracking loops are based on the assumption that the acquisition stage provide a sufficiently small error (otherwise the loop will not converge to the correct path delay), it is hard to make a performance comparison between feedback and feedforward techniques. The feedback techniques are meant to keep the lock, that is, to keep the initial delay estimate as accurate as possible, but once the lock is lost, the acquisition process should be restarted. The feedforward techniques can be seen as one-shot estimates, ${ }^{3}$ which do not need very accurate initial delay estimates in the tracking process (delay errors of the order of chips or tens of chips are possible). For these reasons, the measures of performance are rather dif-

\footnotetext{
${ }^{3}$ When iterative estimates are needed, the same one-shot principle can be applied, by using the previous delay estimates as the starting point when defining the search window for the new delay estimates.
}

ferent in feedback and feedforward algorithms (i.e., for the former, typical measures are the time-to-lose lock and the code tracking noise standard deviation, while for the later, the root-mean-square delay errors and detection probabilities are typically used).

\subsection{Feedforward estimators}

The authors have previously proposed several feedforward delay estimation techniques $[9,30,32,42,43]$ as efficient alternatives to the DLLs-based techniques. These feedforward techniques have been extensively studied for WCDMA signals and BPSK modulation and, among them, the TeagerKaiser (TK) and the deconvolution-based (namely, projection onto convex sets POCS) algorithms proved to be the most promising from the point of view of their performance in closely spaced path scenarios. It is therefore of interest to analyze the behavior of these algorithms in the presence of BOC-modulated PRN codes as well. In what follows, we start from the simplest feedforward estimator, namely, the correlator or matched filter (MF) and then, we present the ideas behind TK and deconvolution-based algorithms.

Based on (8), the MF output at a certain estimated Doppler frequency $\widehat{f_{D}}$ is

$$
J_{\mathrm{MF}}(\tau)=R\left(\widehat{f_{D}}, \tau\right)
$$

The estimate of the Doppler frequency $\widehat{f_{D}}$ is obtained as the frequency corresponding to the global maximum of the time-frequency mesh illustrated in Figure 4. We remark that, for a fair comparison, the same $\widehat{f_{D}}$ estimated (based on MF output) is kept for all the compared delay estimators; only the delay estimation process is different. By taking the discrete samples $\tau=l T_{s}$ of the MF output of (11), we can rewrite the MF output in a vectorial form [30] (needed to explain the deconvolution algorithms):

$$
\mathbf{J}_{\mathrm{MF}}=\mathbf{G}_{\mathrm{BOC}} \mathbf{h}+\mathbf{v}
$$

where $\mathbf{J}_{\mathrm{MF}}=\left[J_{\mathrm{MF}}\left(d_{\min } T_{s}\right), \ldots, J_{\mathrm{MF}}\left(d_{\max } T_{s}\right)\right]^{T}, d_{\min }$ is the minimum delay in samples, and $d_{\max }$ is the maximum delay in samples (i.e., the time-window or the delay spread over which we look for the channel paths spans between $d_{\min } T_{s}$ and $d_{\max } T_{s}$ seconds, and $d_{\min }$ and $d_{\max }$ are chosen as integer multiples of the sampling period, for the sake of the simulation model), the sampling interval $T_{s}$ is chosen sufficiently small to model fractional path delays ${ }^{4}$ (e.g., $T_{s}=0.05 T_{\mathrm{BOC}}$ ).

We remark that, similarly with feedback techniques, $d_{\text {min }}$ and $d_{\max }$ can be chosen in such a way to capture the channel true delays, based on previous delay estimates or based on the acquisition stage. For example, for diminishing the number

\footnotetext{
4 The fractional delays model and the estimation of the delays with high accuracy can be achieved either via a sufficiently small sampling interval (i.e., a high number of samples per chip), or, equivalently, via interpolation. Interpolation-based algorithms may decrease the receiver complexity and constitutes a topic of future research.
} 
of correlators required by the model, an initial acquisition stage can take place (where a coarse delay estimate $\hat{\tau}_{\text {LOS }}$ is formed), then the feedforward-based fine delay estimation stage will perform the correlations only $\pm D_{\max } / 2$ chips around $\hat{\tau}_{\text {LOS }}$, where $D_{\max }$ is the search window length in chips (i.e., $d_{\min }=\left(\hat{\tau}_{\mathrm{LOS}}-D_{\max } / 2\right) N_{s} N_{\mathrm{BOC}}$ and $d_{\max }=\left(\hat{\tau}_{\mathrm{LOS}}+\right.$ $\left.\left.D_{\max } / 2\right) N_{s} N_{\text {BOC }}\right)$. For feedback tracking techniques, the LOS delay is typically tracked within \pm 1 chip around the previous delay estimate, while in our case, we can have $D_{\max }>2$ chips (indeed, in our simulation we used a $D_{\max }$ between 4 and 10 chips).

Above, $\mathbf{G}_{\mathrm{BOC}}$ is the ideal autocorrelation matrix of size $N \times N\left(N=d_{\max }-d_{\min }\right)$, including the effect of BOC modulation and having the elements $g(i, j)=\mathcal{R}_{\mathrm{BOC}}((i-$ j) $\left.T_{s}\right), i, j=1, \ldots, N$, and $\mathbf{h}$ is a $N \times 1$ vector, including the channel effect and having the $i$ th element equal to $\sqrt{E_{b}} e^{j \pi \Delta f_{D} N_{c} T} \operatorname{sinc}\left(\pi \Delta f_{D} N_{c} T\right) h_{i}, i=d_{\min }, \ldots, d_{\max }, \Delta f_{D}=$ $f_{D}-\widehat{f_{D}}$, and

$\bar{h}_{i}= \begin{cases}\tilde{\alpha}_{i} & \text { if a channel path is present at the time delay } i T_{s} \\ 0 & \text { otherwise. }\end{cases}$

The term $\mathbf{v}$ is the noise vector, with the elements $\tilde{\eta}\left(\widehat{f_{D}}, i T_{s}\right)$ (including various noise sources such as the background noise, the nonidealities of the PRN code sequences, the possible interference between two or more satellites, etc.), $i=$ $d_{\min }, \ldots, d_{\max }$. The MF estimate of the squared channel coefficient envelope $|\mathbf{h}|^{2}$ is given by the noncoherently averaged MF output:

$$
\widehat{\mathbf{h}}_{\mathrm{MF}}=\frac{1}{N_{n c}} \sum_{1}^{N_{n c}}\left|\mathbf{J}_{\mathrm{MF}}\right|^{2}
$$

where $N_{n c}$ is the noncoherent integration time. In what follows, we will refer to $\widehat{\mathbf{h}}$ estimates also as "cost functions." Simulation results showed that using the squaring-absolute value operator (instead of the absolute value itself) gives slightly better results. The noncoherent squaring losses are indeed present, but noncoherent averaging might still be needed, due to the limits in the coherent integration (e.g., residual Doppler shifts, instabilities of oscillator clock, etc.)

Resolving the multipath components can be seen as a deconvolution problem [30] in which we try to estimate the nonzero elements of the unknown gain vector $\mathbf{h}$. The first nonzero component higher than a threshold will be the estimate of the first arriving path. [9]

The well-known least squares (LS) solution is given by

$$
\widehat{\mathbf{h}}_{\mathrm{LS}}=\left(\mathbf{G}_{\mathrm{BOC}}^{H} \mathbf{G}_{\mathrm{BOC}}\right)^{-1} \mathbf{G}_{\mathrm{BOC}}^{H} \widehat{\mathbf{h}}_{\mathrm{MF}} .
$$

We remark that the above LS solutions also suffer of noncoherent losses, due to the fact that we use $\widehat{\mathbf{h}}_{\mathrm{MF}}$ in the estimator, instead of $\mathbf{J}_{\mathrm{MF}}$. Thus, the noise statistics are modified (to a chi-square distribution), and the LS solution becomes suboptimal. However, due the practical limits of coherent integration mentioned above, the noncoherent squaring should be usually employed. Indeed, simulation results with even a small residual Doppler shifts showed that, by using coherent integration alone, we cannot achieve satisfactory results. The solution given by (15) is known to be very sensitive to noise and often the matrix $\mathbf{G}_{\mathrm{BOC}}^{H} \mathbf{G}_{\mathrm{BOC}}$ is ill-conditioned. It will be kept in what follows as a reference, but the results will be shown to be very poor, as expected. More robustness to the noise is given by the so-called minimum mean square error (MMSE) solution, given by

$$
\widehat{\mathbf{h}}_{\mathrm{MMSE}}=\left(\hat{\sigma}^{2} \mathbf{I}+\mathbf{G}_{\mathrm{BOC}}^{H} \mathbf{G}_{\mathrm{BOC}}\right)^{-1} \mathbf{G}_{\mathrm{BOC}}^{H} \widehat{\mathbf{h}}_{\mathrm{MF}},
$$

where $\mathbf{I}$ is the unity matrix and $\hat{\sigma}^{2}$ is the estimate of the noise variance, obtained directly from the MF output $\widehat{\mathbf{h}}_{\mathrm{MF}}$, as it will be discussed in Section 3.3.

In order to cope with the noise in even a better way and in order to solve the problem of closely spaced paths, the MMSE solution can be developed into a constrained iterative deconvolution technique, called projection onto convex sets (POCS), which was introduced in $[33,44]$, for the Rake receiver with rectangular pulse shapes, and later applied for WCDMA signals $[9,30]$. The POCS algorithm is an iterative method that finds a feasible solution consistent with a number of constraints [12]. Starting with an initial guess of the solution, the algorithm converges to a feasible solution by cyclically projecting into constraint sets. Thus, POCS estimator of $\mathbf{h}$ has the form $\widehat{\mathbf{h}}_{\text {POCS }}=\mathcal{P}_{\mathcal{C}} \mathbf{h}$, where $\mathcal{P}_{C}(\cdot)$ is the projection operator and $C$ is the convex set defined by the MF output: $C=\left\{\mathbf{f},\left\|\mathbf{J}_{\mathrm{MF}}-\mathbf{G}_{\mathrm{BOC}} \mathbf{f}\right\|^{2} \leq \xi\right\}[33,44]$ where $\|\cdot\|$ is the $L 2$ vector norm (i.e., by definition, if $z$ is a column vector, its $L 2$ norm is $\|z\|^{2}=z^{h} z$ ), and $\xi$ is a scalar bound, given by the variance of the noise at the output of MF. The POCS solution is found by solving the following quadratic program [43]:

$$
\left\{\begin{array}{c}
\min \left\|\hat{\mathbf{h}}_{\text {POCS }}-|\mathbf{h}|^{2}\right\|^{2}, \\
\text { under the constraint: }\left\|\mathbf{J}_{\mathrm{MF}}-\mathbf{G}_{\mathrm{BOC}} \mathbf{h}\right\|^{2} \leq \xi
\end{array}\right\} .
$$

The squaring of the channel vector $\mathbf{h}$ in the above equation was necessary because the $\hat{\mathbf{h}}$ estimates given here (for all the algorithms) are, in fact, the estimates of $|\mathbf{h}|^{2}$ (and not of the channel coefficient vector $\mathbf{h}$ ). This fact does not have any impact on the delay estimates, since we are not interested in the exact values of the channel coefficients, but only on their relative magnitudes (i.e., we are interested in finding those values of estimated vectors $\widehat{\mathbf{h}}$ which are higher than a certain threshold).

The above quadratic program can be solved iteratively and POCS estimation can take place in several stages. At stage $k+1$, the POCS estimate can be written as $[12,30,43]$

$$
\begin{aligned}
\hat{\mathbf{h}}_{\mathrm{POCS}}^{(k+1)}= & \hat{\mathbf{h}}_{\mathrm{POCS}}^{(k)}+\left(\frac{1}{\lambda} \mathbf{I}+\mathbf{G}_{\mathrm{BOC}}^{H} \mathbf{G}_{\mathrm{BOC}}\right)^{-1} \\
& \times \mathbf{G}_{\mathrm{BOC}}^{H}\left(\widehat{\mathbf{h}}_{\mathrm{MF}}-\mathbf{G}_{\mathrm{BOC}} \hat{\mathbf{h}}_{\mathrm{POCS}}^{(k)}\right),
\end{aligned}
$$




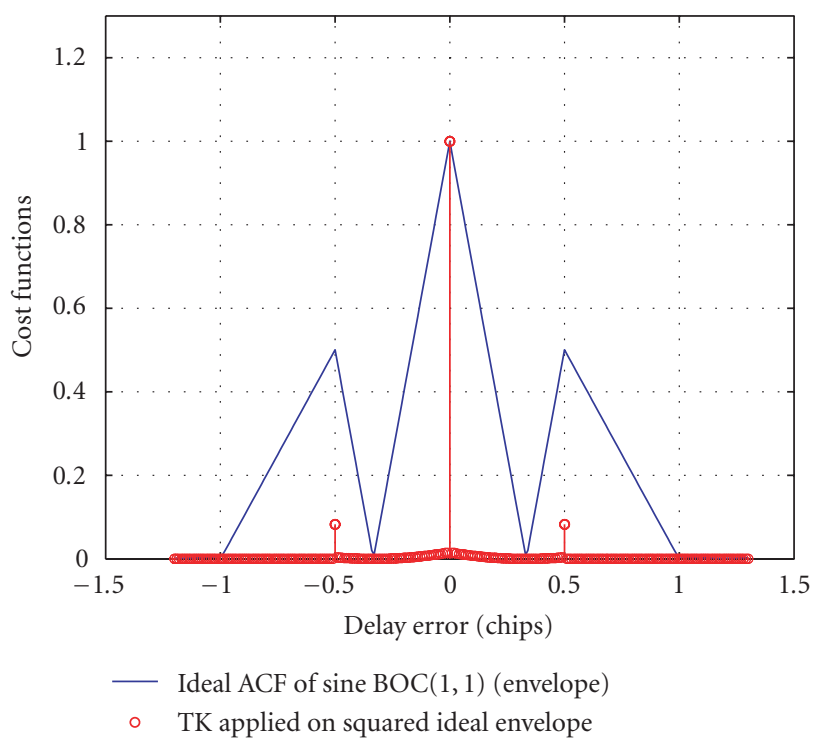

FIGURE 7: Illustration of TK applied on the squared envelope of an ideal ACF of sine $\operatorname{BOC}(1,1)$ signal (no noise).

where $\lambda$ is a constant determining the convergence speed (it also represents the Lagrange multiplier associated with the constraint of (17)). The initial estimate for $\hat{\mathbf{h}}_{\text {POCS }}$ is the MF estimate: $\widehat{\mathbf{h}}_{\text {POCS }}^{(1)}=\widehat{\mathbf{h}}_{\mathrm{MF}}$. The final cost function for POCS estimation is $\widehat{\mathbf{h}}_{\text {POCS }}=\widehat{\mathbf{h}}_{\text {POCS }}^{\left(N_{\text {iter }}\right)}$.

In practice, iterations are performed until no significant improvement from iteration to iteration is achieved. Optimally, $\lambda$ should be adjusted based on the noise variance and the other bounds in the optimization process [12, 14, 45]; however, this adjustment is a laborious process, based on a priori knowledge of noise statistics (which, in practice, might be unknown). Moreover, the simulation results with various $\lambda$ values between 0.01 and 10 showed us that the variation of $\lambda$ does not have a significant impact on the delay estimation accuracy and that choosing $\lambda \in[0.1,1]$ slightly outperforms the cases when $\lambda>1$ (thus, $\lambda=0.5$ is a reasonable choice). Also based on simulations, we noticed that we need at least $N_{\text {iter }}=10$ iterations in order to be able to separate the closely spaced paths, which is also in accordance with the results reported in [14].

We remark that the notion of closely spaced paths refers usually to paths separated at less than one chip interval $[7,9-$ 16]. However, due to the narrower width of the main lobe of the ACF in the presence of BOC modulation (as seen in Figure 3), the most challenging cases will be in fact those with a path separation of less than $1 /\left(N_{\text {BOC }}\right)$ chips, as it will be seen from the simulation results.

The nonlinear quadratic TK operator was first introduced for measuring the real physical energy of a system [46]. Since its introduction, it has widely been used in various speech processing and image processing applications and, more recently, it has also been applied in CDMA applications $[9,30,34,35,42]$. The discrete-time TK operator $\Psi_{d}(\cdot)$ of a complex-valued discrete signal $z(n)$ is $[9,42]$

$$
\Psi_{d}(z(n)) \triangleq z^{2}(n-1)-\frac{1}{2}\left(z(n-2) z^{*}(n)+z(n) z^{*}(n-2)\right),
$$

and the discrete-time TK operator $\Psi_{d}(\cdot)$ of a real-valued discrete signal $z(n)$ becomes

$$
\Psi_{d}(z(n)) \triangleq z(n-1) z^{*}(n-1)-z(n-2) z(n) .
$$

In our case, TK operator is applied on the squared-absolute value of the MF output, and the cost function for TK algorithm (after noncoherent averaging) is

$$
\widehat{\mathbf{h}}_{\mathrm{TK}}=\left|\Psi_{d}\left(\left|\hat{\mathbf{h}}_{\mathrm{MF}}\right|^{2}\right)\right| \text {. }
$$

The reason for choosing TK operator in the algorithm comparison is its good performance reported in multipath scenarios for WCDMA systems [9, 30, 42]. We remark that TK operator was first applied at different levels of the correlation function: before coherent integration, before noncoherent integration, and after both coherent and noncoherent integration. The results showed that the best results are obtained when TK is applied after noncoherent integration (and therefore, on the squared-absolute value of the averaged correlation function), as shown in (21), and the results are only shown for this case. For the other situations (i.e., TK applied before integration), the results are quite poor, due to the high noise levels and to the sensitivity of TK operator to the noise. The intuitive behavior of TK algorithm is illustrated via Figure 7, where we show the envelope of a sine BOC $(1,1)$ signal (continuous line) together with the output of TK operator applied on the squared envelope of the ACF. We notice that TK is able to distinguish the global peak (corresponding to the zero delay error) among the spurious sidelobes of the sine-BOC ACF. The side-lobes are not completely cancelled out after applying TK operator, but their levels are much diminished after TK. This property of TK to preserve only the useful energy of the correlation function will be indeed beneficial for closely spaced channel paths (see later on the explanations with respect to Figure 9).

In Figures 8 and 9 we illustrate the performance of POCS and TK, respectively, in the presence of 4 closely spaced paths and BOC-modulated PRN codes (the noiseless case is shown here). A scenario with LOS path weaker than a successive NLOS component was selected for illustrative purposes. The same channel profile as that one used for Figure 6 is also used here. Typically, better results are achieved when LOS path is the strongest one. The true channel path delays are plotted with their respective magnitudes for reference purposes. From the matched filter output, we cannot distinguish the presence of multipath components. If the estimation is based on MF output, the delay estimation error would be 0.05 chips (which translates into about $14.6 \mathrm{~m}$ distance error for a chip rate of $1.023 \mathrm{MHz}$ ). By applying TK operator (Figure 9), all the four channel paths are easily distinguished. POCS estimates (Figure 8) are a little bit noisier, but they are still estimating the LOS delay better than MF (in this example, the delay error for the first path is 0.02 chips or $5.86 \mathrm{~m}$ ). 


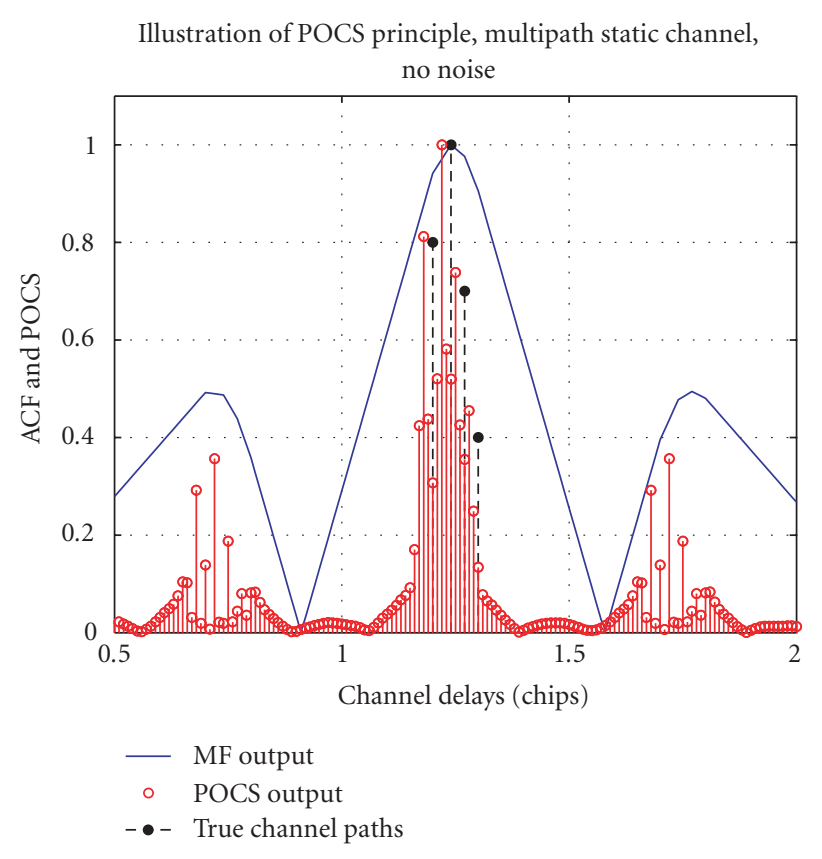

FIGURE 8: Illustration of POCS delay estimation algorithm in the presence of $\operatorname{BOC}(2,2)$ or $\operatorname{BOC}(1,1)$ modulation $\left(N_{\mathrm{BOC}}=2\right)$ and 4 closely spaced paths.

\subsection{Threshold setting}

As explained above, a threshold is necessary to be set in order to select the first significant local maximum of the cost function $\hat{\mathbf{h}}$ (e.g., $\widehat{\mathbf{h}}_{\mathrm{MF}}, \widehat{\mathbf{h}}_{\mathrm{TK}}, \widehat{\mathbf{h}}_{\mathrm{POCS}}$, etc.). The time position of the channel paths is determined as the position of the local peaks of the cost function which are higher than a threshold $\gamma$. This threshold was built based on the ideal ACF of BOCmodulated signal together with the estimate of the noise variance:

$$
\gamma=\gamma_{1}+\sqrt{\hat{\sigma}^{2}}
$$

where $\gamma_{1}$ is the second highest peak of an ideal ACF in the presence of BOC modulation (e.g., as seen in Figure 7, $\gamma_{1}=0.5$ for $N_{\mathrm{BOC}}=2$ ), and $\hat{\sigma}^{2}$ is the estimate of the noise variance, obtained directly from the cost function $\widehat{\mathbf{h}}_{\text {alg }}$ as the mean of the squares of out-of-peak values of $\hat{\mathbf{h}}_{\text {alg }}$. An out-ofpeak (OOP) value is a value which is at least one chip apart from the global peak and alg stands for one of the MF, LS, MMSE, POCS, or TK algorithms:

$$
\hat{\sigma}^{2}=\frac{1}{N_{\mathrm{OOP}}} \sum_{n \in \text { indices of OOP values }}\left|\hat{h}_{\mathrm{alg}}(n)\right|^{2} .
$$

Above, $N_{\text {OOP }}$ is the number of discrete OOP samples and $\hat{h}_{\text {alg }}(n)$ are the elements of the $\hat{\mathbf{h}}_{\text {alg vectors. Equation (22) has }}$ been used for MF, POCS, MMSE, and LS estimates. For TK algorithm, $\gamma_{1}$ is obtained directly from the TK applied on the square envelope of an ideal ACF (see Figure 7), and the noise variance is obtained directly from the MF output. An example for the threshold computation for MF and TK outputs is

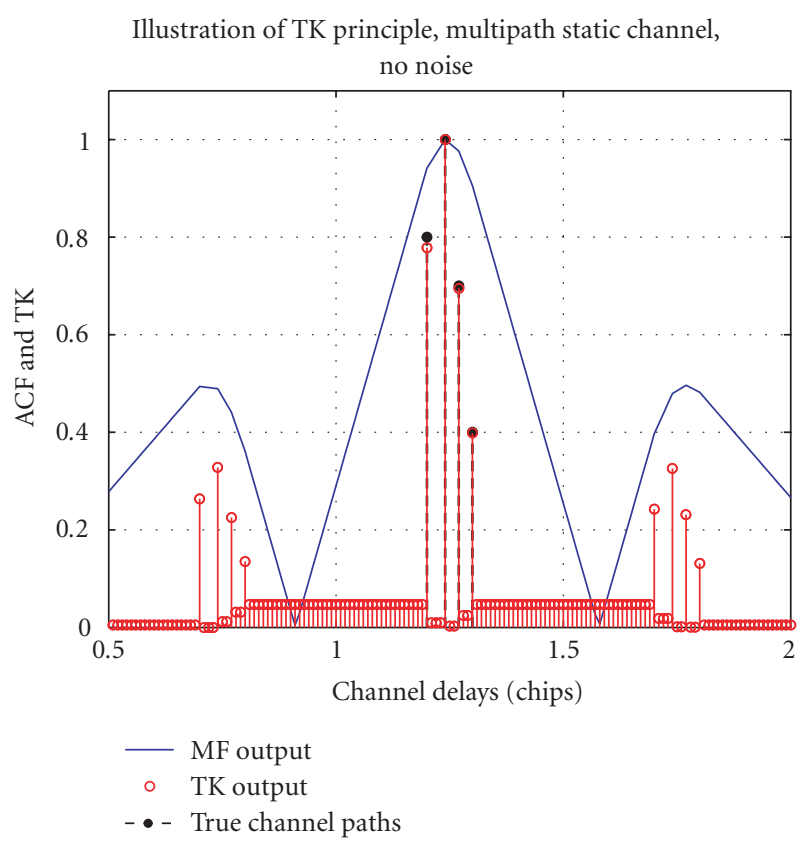

FIGURE 9: Illustration of TK delay estimation algorithm in the presence of $\operatorname{BOC}(2,2)$ or $\operatorname{BOC}(1,1)$ modulation $\left(N_{\mathrm{BOC}}=2\right)$ and 4 closely spaced paths.

shown in Figure 10 for a 4-path fading channel and CNR of $27 \mathrm{~dB}-\mathrm{Hz}$. The true LOS delay and the estimated LOS delay are also written in each plot.

We also remark here that the side-lobes of a sine BOCmodulated signal appear at the delays $\tau_{\text {sidelobes, }}$ given by

$$
\tau_{\text {sidelobes }}=\arg \max _{\tau} \mathcal{R}_{\mathrm{BOC}}(\tau)
$$

with $\mathcal{R}_{\mathrm{BOC}}(\tau)$ given in (9). For example, the side peaks for sine $\operatorname{BOC}(1,1)$ modulation $\left(N_{\mathrm{BOC}}=2\right)$ occur at \pm 0.5 chips around the global maximum, for sine $\operatorname{BOC}(15,10)\left(N_{\mathrm{BOC}}=\right.$ 3 ) occur at \pm 0.33 and \pm 0.67 chips, and for sine $\operatorname{BOC}(10,5)$ $\left(N_{\mathrm{BOC}}=4\right)$ occur at $\pm 0.25, \pm 0.5$, and \pm 0.75 chips. Generally, there are $2 N_{\mathrm{BOC}}-2$ side-lobes in the correlation function which interfere with the channel paths and may create false lock points. However, the most significant ones are those with the smallest delay relative to the global maximum. This is the reason for which the threshold estimation is based on the second highest peak of the ideal ACF given in (9).

\section{PERFORMANCE COMPARISON}

In what follows, the performance of the discussed feedforward delay estimation algorithms is compared in terms of detection probability $P_{d}$ and root-mean-square error (RMSE). The reason for not including the feedback delay estimation algorithms in this comparison is that there is no possibility of a fair comparison between the two. This comes from the fact that the performance measure for feedback-based algorithms is typically the time-to-lose lock, which has no equivalent for the feedforward-based algorithms. Moreover, 


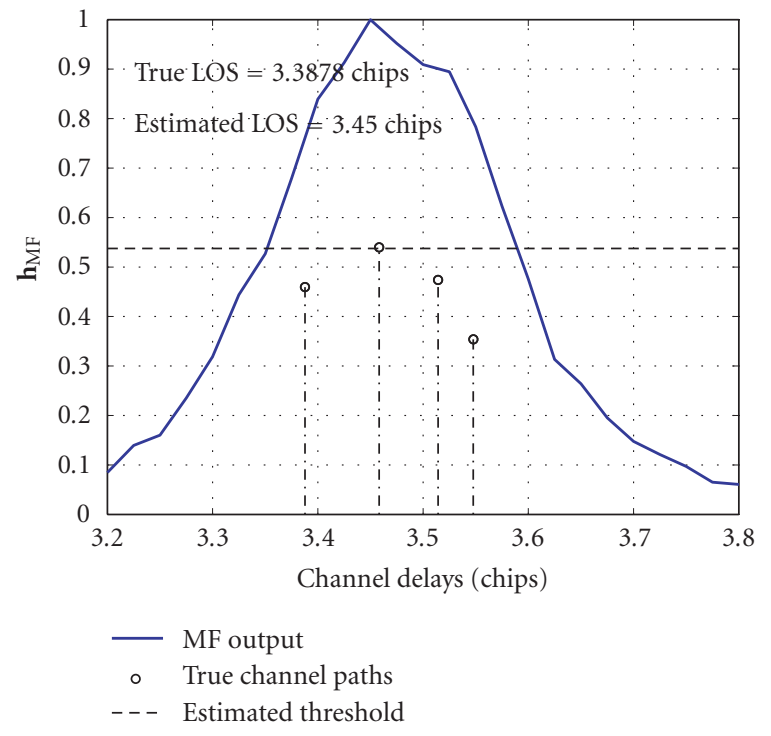

(a) Estimated threshold: $\gamma=0.53763$

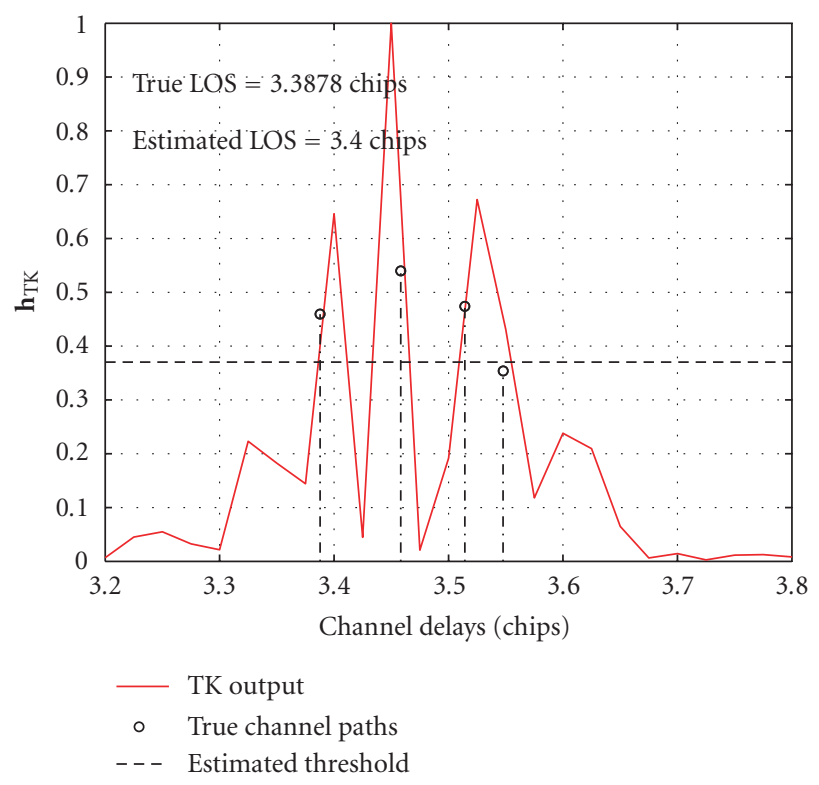

(b) Estimated threshold: $\gamma=0.37032$

Figure 10: MF and TK outputs (main lobe) for a 4-path fading channel and the estimation of the threshold, $N_{\mathrm{BOC}}=2, \mathrm{CNR}=$ $27 \mathrm{~dB}-\mathrm{Hz}, N_{c}=180, N_{n c}=8$.

in feedback-based algorithms, we have to assume that the initial delay error is less than $1 /\left(2 N_{\mathrm{BOC}}\right)$ chips in order for the algorithm to converge (which is a very restrictive assumption).

The performance of the algorithms for channel profiles has been analyzed and the most representative results have been included. Two main channel profiles have been considered (both may be seen as typical indoor channels, due to large number of closely spaced paths and low mobile speeds):
TABle 2: Parameters of the simulations.

\begin{tabular}{ll}
\hline$N_{\text {BOC }}$ & BOC modulation order \\
$N_{c}$ & Coherent integration time (ms) \\
$N_{n c}$ & $\begin{array}{l}\text { Noncoherent integration time } \\
\text { (blocks of } N_{c} \text { ms) }\end{array}$ \\
$v$ & Mobile receiver speed (km/h) \\
& Maximum separation between successive \\
$x_{\max }$ & paths (chips) \\
$\bar{\alpha}$ & Vector of average path powers (dB) \\
$L$ & Number of channel paths (if constant) \\
$L_{\max }$ & $\begin{array}{l}\text { Maximum number of channel paths } \\
\text { (if random) }\end{array}$ \\
$\mu_{\mathrm{PDP}}$ & $\begin{array}{l}\text { Exponential factor for the decaying } \\
\text { PDP model (chips }\end{array}$ \\
& $\begin{array}{l}\text { The error for which the detection probability } \\
\text { is computed (chips), that is, detection is done } \\
\Delta \varepsilon_{P_{d}}\end{array}$ \\
\end{tabular}

(i) indoor with Rayleigh distribution of all paths, decaying power delay profile (PDP) and a random number of paths, uniformly distributed between 1 and $L_{\max }=$ 7 ,

(ii) indoor with fixed Rayleigh PDP (first path having a smaller average power than the second one) and $L=4$ paths.

The mobile speed was set to $v=4 \mathrm{~km} / \mathrm{h}$ (we remark that simulations with higher mobile speeds and with Rician fading profiles have also been performed and similar conclusions were drawn). The channel models used here are based on some typical fading channel models reported in the literature $[9,40,47]$. A main parameter of the channel model is the separation between successive paths, which was assumed to be uniformly distributed between 0 and $x_{\max }$ (where $x_{\max }$ is the maximum separation between successive paths).

When the decaying PDP is used, the average path power $\bar{\alpha}_{l}$ of the $l$ th path is given according to its distance from the first arriving path and to an exponential factor $\mu_{\mathrm{PDP}}$ in the form $\bar{\alpha}_{l}=\bar{\alpha}_{1} e^{\mu_{\mathrm{PDP}}\left(\tau_{l}-\tau_{1}\right)}$.

The detection probability $P_{d}$ is defined as the probability to detect the first arriving path (hereby assumed to be LOS path) with an absolute error smaller than or equal to $\Delta \varepsilon_{P_{d}}$. The LOS delay estimation is done only at the correct frequency bin (with a possible small residual Doppler error, smaller than $1 / N_{c} \mathrm{KHz}$ ), and with a time-window $D_{\max }$, as seen in Section 3.2. The main parameters of the simulation model are summarized in Table 2 and their values are given in the caption of each figure.

The comparison between the MF, TK, POCS, and LS algorithms for various channel profiles is shown in Figures 11 and 12 (the plots versus CNR), in Figure 14 (the plots versus $N_{\mathrm{BOC}}$ ), and in Figure 15 (the plots versus $N_{c}$ ). Clearly, LS algorithm fails to work properly due to the noise enhancement property specific to LS approaches. MMSE algorithms 


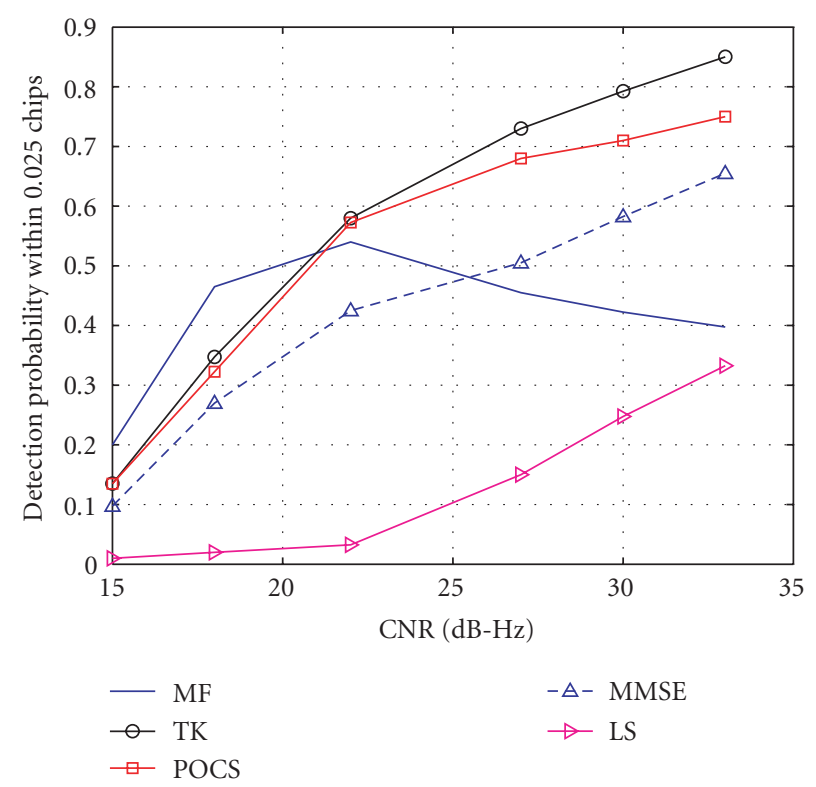

(a) Indoor channel, decaying PDP, $x_{\max }=0.1$ chips, $L_{\max }=7$ paths

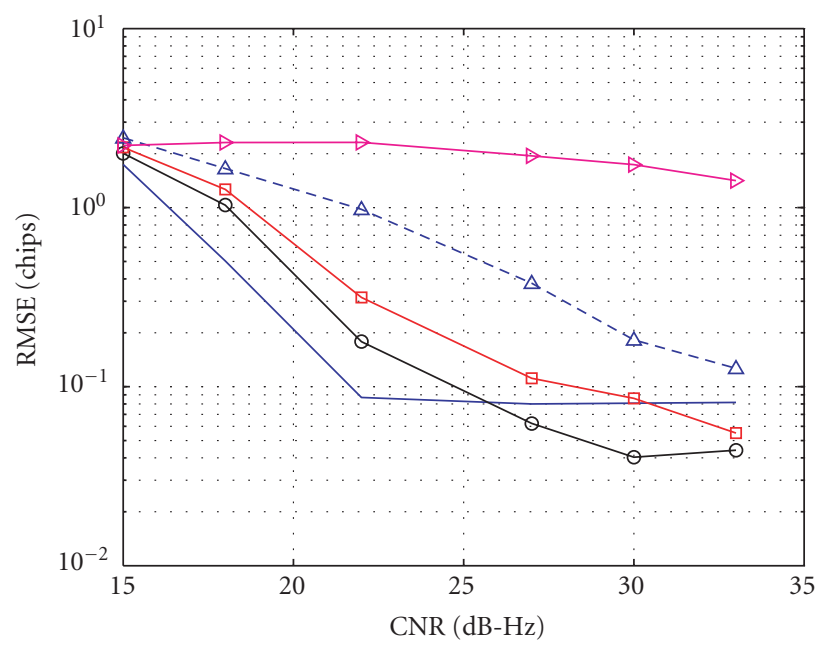

$$
\begin{array}{ll}
- \text { MF } & -\triangle \text { - MMSE } \\
- \text { TK } & \rightarrow \text { LS } \\
\square \text { POCS } &
\end{array}
$$

(b) Indoor channel, decaying PDP, $x_{\max }=0.1$ chips, $L_{\max }=7$ paths

FIgURE 11: Comparison of feedforward delay estimation algorithms as a function of CNR, indoor channel, decaying PDP, $\mu_{\mathrm{PDP}}=0.5$, $x_{\max }=0.1$ chips, $N_{c}=180, N_{n c}=8, N_{\mathrm{BOC}}=2, L_{\max }=7$ paths, $v=4 \mathrm{~km} / \mathrm{h} . P_{d}$ within $\Delta \varepsilon_{P_{d}}=0.025$ chips error (a) and RMSE in chips (b).

is better than LS, but it is still surpassed by TK and POCS, and, in some cases, even by MF; one reason might be the fact that MMSE is using the estimated noise variance, and not the true noise variance (which is hard to get in practice), and therefore, it might be affected by the errors in this estimate.

We noticed from Figures 11 and 12 that MF algorithm is not able to distinguish well between very closely spaced
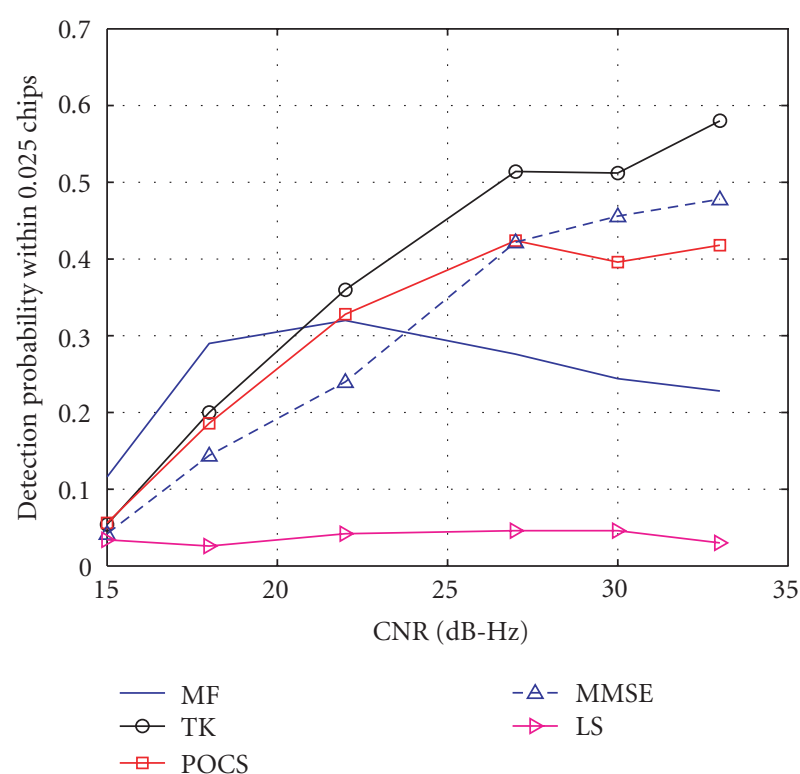

(a) Indoor channel, fixed PDP, $x_{\max }=0.2$ chips, $L=4$ paths

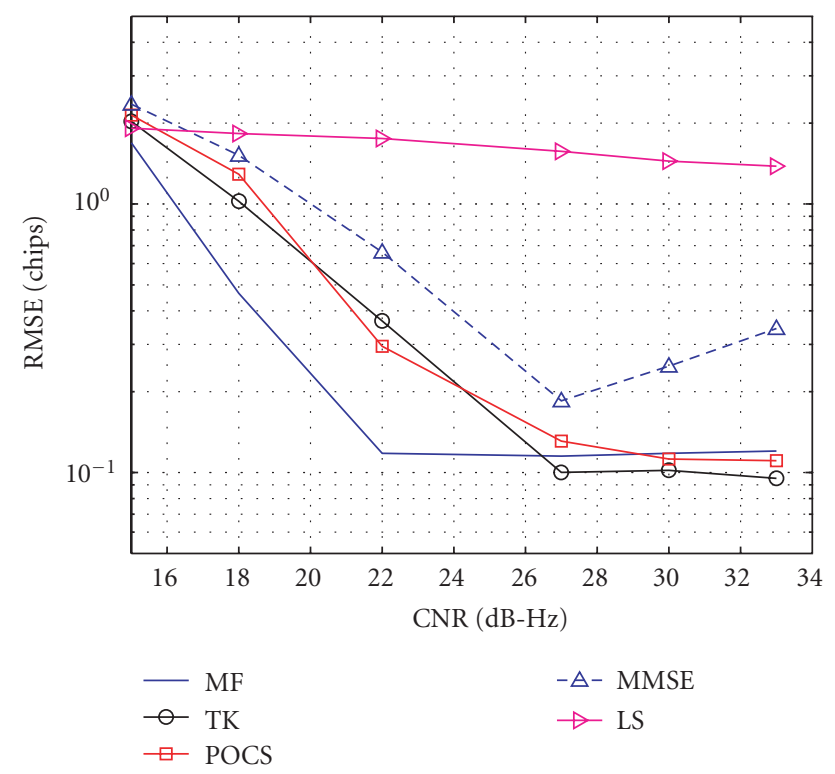

(b) Indoor channel, fixed PDP, $x_{\max }=0.2$ chips, $L=4$ paths

FIgURE 12: Comparison of feedforward delay estimation algorithms as a function of CNR, indoor channel, fixed PDP: $\bar{\alpha}=[-2,0,-1,-4] \mathrm{dB}, x_{\max }=0.2$ chips, $L=4$ paths, $N_{c}=180$, $N_{n c}=8, N_{\text {BOC }}=2, v=4 \mathrm{~km} / \mathrm{h} . P_{d}$ within $\Delta \varepsilon_{P_{d}}=0.025$ chips error (a) and RMSE in chips (b).

paths (i.e., maximum spacing less than 0.2 chips), and therefore, it suffers from a saturation effect at higher CNRs (see the $P_{d}$ curves in the above-mentioned plots). Both TK and POCS have much better detection probability than MF algorithms if the CNR is sufficiently high (or, equivalently, if we use enough integration to smooth the signal). This is due to the fact that TK and POCS can separate closely spaced paths, 


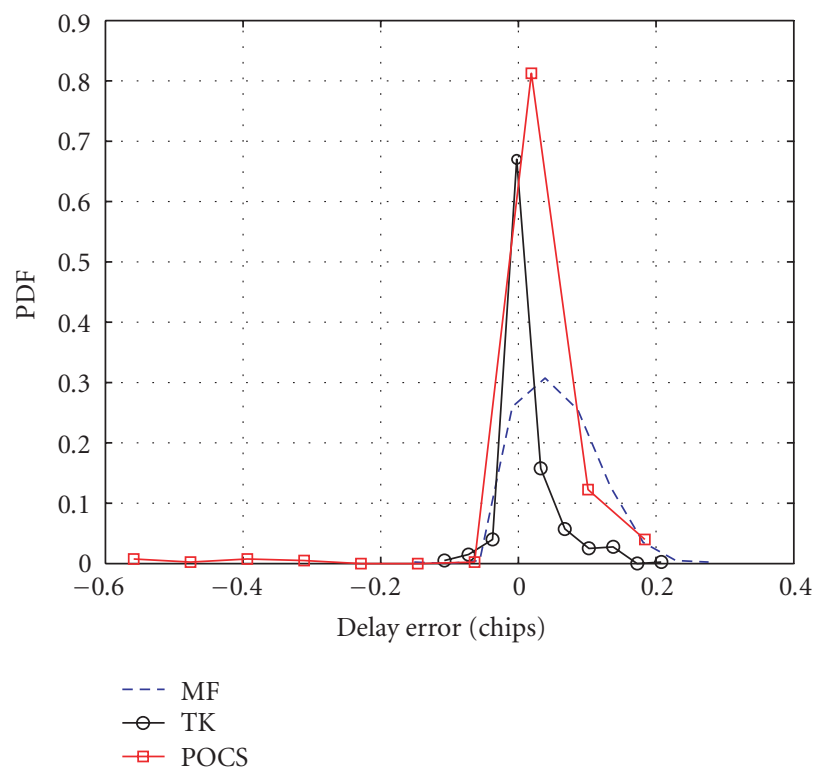

FIGURE 13: Distribution of delay estimation errors, indoor channel, decaying PDP, $\mu_{\mathrm{PDP}}=0.5, x_{\max }=0.1$ chips, $N_{c}=180, N_{n c}=8$, $N_{\text {BOC }}=2, L_{\max }=7$ paths, $v=4 \mathrm{~km} / \mathrm{h}, \mathrm{CNR}=30 \mathrm{~dB}-\mathrm{Hz}$.

while the MF will always detect the merged peak, as shown in Figures 9, 8, and 10.

On the other hand, from the point of the RMSE value, MF is not much worse than TK and POCS at high CNR values and it is always better in severe noise conditions (low CNR). This means that, when the estimate is noisy in TK and POCS cases, this estimate is more likely to be an outlier, while for MF, it mostly remains in a neighborhood of the true path delays, but without being able to separate them. The apparent contradiction between a good $P_{d}$ and a rather poor RMSE is illustrated in Figure 13 via the probability distribution function (PDF) of the delay errors. This plot corresponds to the $\mathrm{CNR}=30 \mathrm{~dB}-\mathrm{Hz}$ from Figure 11, where we notice that $P_{d}$ of TK and POCS is much better than the $P_{d}$ of MF, while the gap between the RMSE of TK and the RMSE of MF is not very high, and POCS has even worse RMSE performance than MF. If we look at the PDF of Figure 13, we see that MF estimate has a higher bias than the other two estimates (due to the incapacity of MF to separate closely spaced paths), but it also has less outliers.

We remark that, when we loosen the condition for the allowed delay error $\Delta \varepsilon_{P_{d}}$ (i.e., $\Delta \varepsilon_{P_{d}}$ increases), the detection probability becomes better, as expected, but the general shapes of the curves are preserved.

As seen in Figures 11 and 12, the behavior of the compared algorithms is pretty similar in decaying PDP channels, as well as in fixed PDP channels. However, if the first arriving path is weaker than the next arriving path, as in Figure 12, the detection probability decreases for all the algorithms, and MF is clearly not good enough to detect the first arriving peak (neither in detection probability nor in RMSE).

From the comparison between different algorithms in various channel profiles, we noticed that TK and POCS es-

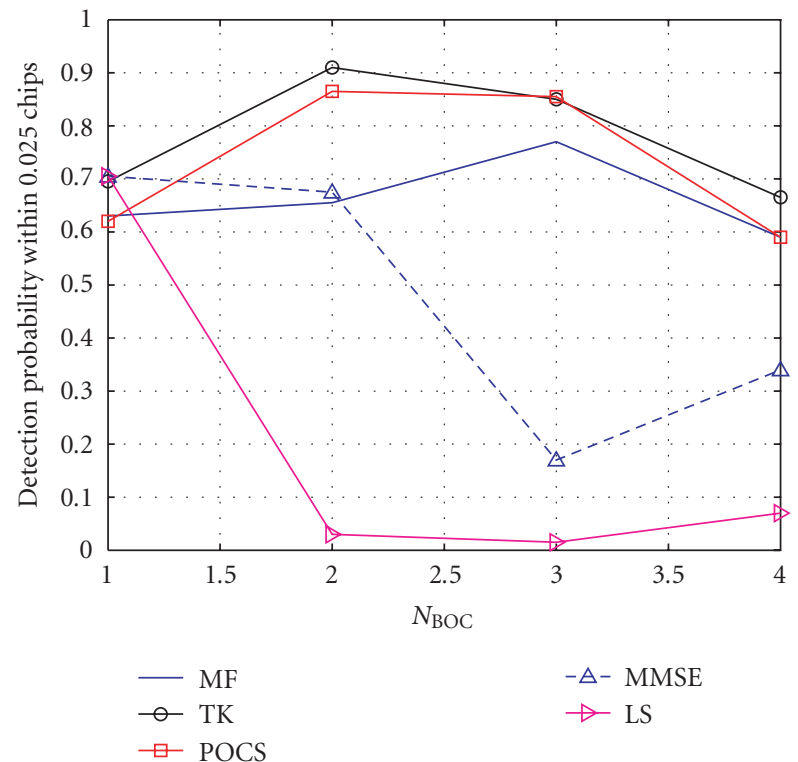

(a) Indoor channel, decaying PDP, $x_{\max }=0.05$ chips, $L_{\max }=7$ paths

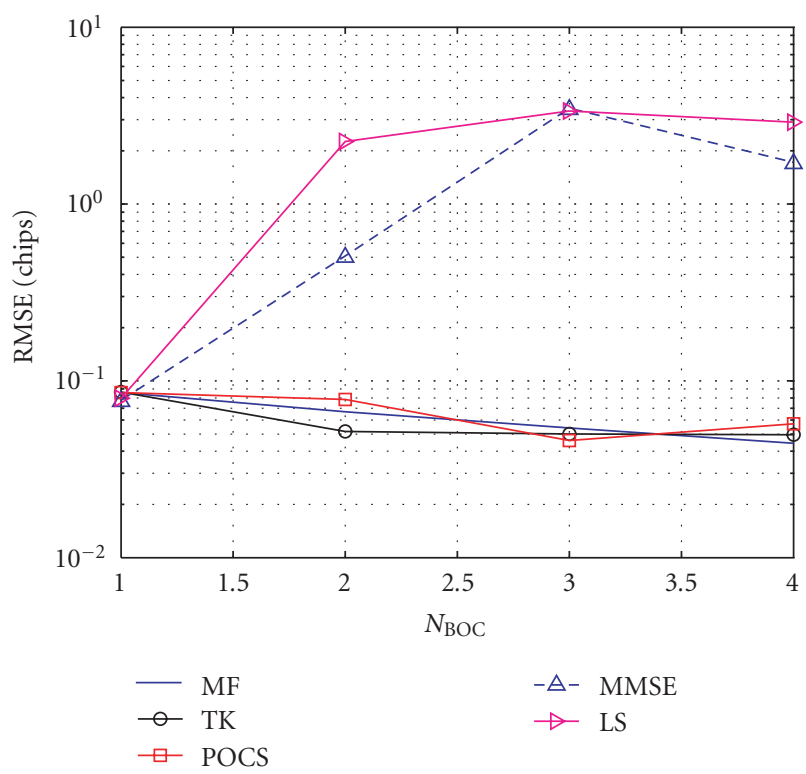

(b) Indoor channel, decaying PDP, $x_{\max }=0.05$ chips, $L_{\max }=7$ paths

FIGURE 14: Comparison of feedforward delay estimation algorithms as a function of $N_{\mathrm{BOC}}$, indoor channel, decaying PDP, $x_{\max }=$ 0.05 chips, $N_{c}=180, N_{n c}=8, C N R=30 \mathrm{~dB}-\mathrm{Hz}, v=4 \mathrm{~km} / \mathrm{h}$. $P_{d}$ within $\Delta \varepsilon_{P_{d}}=0.025$ chips error (a) and RMSE in chips (b).

timators are less robust to noise than MF estimator. This is partially also due to the threshold computation $\gamma$, which is quite noisy in low CNR conditions, and therefore increases the likelihood of picking a wrong local peak of the correlation function as the LOS estimate. On the other hand, if CNR after integration is sufficiently high, we notice that TK and POCS offer the best separation between closely spaced 


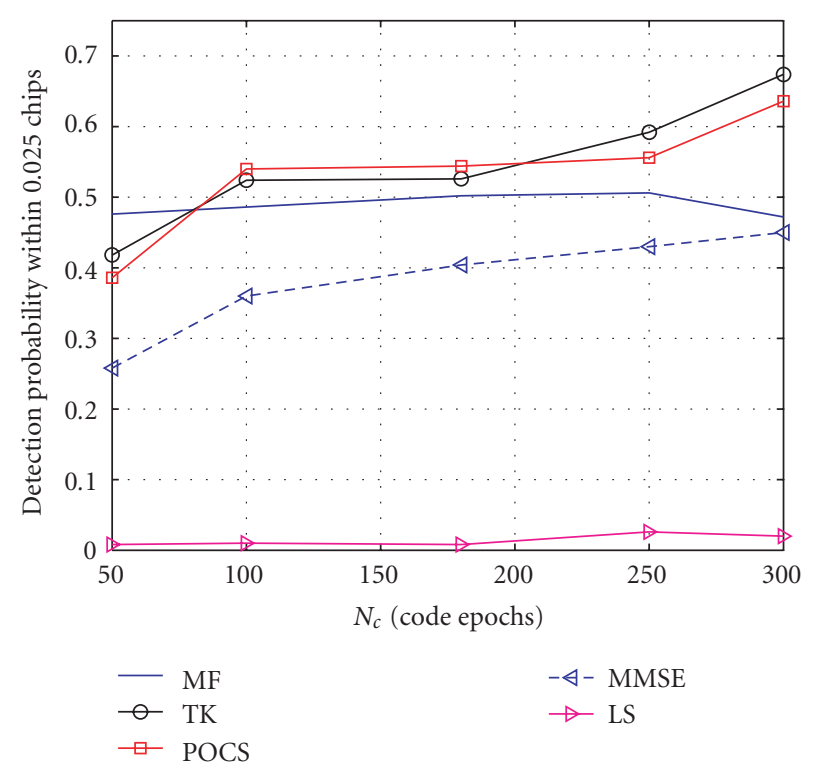

(a) Indoor channel, decaying PDP, $x_{\max }=0.1$ chips, $L_{\max }=7$ paths

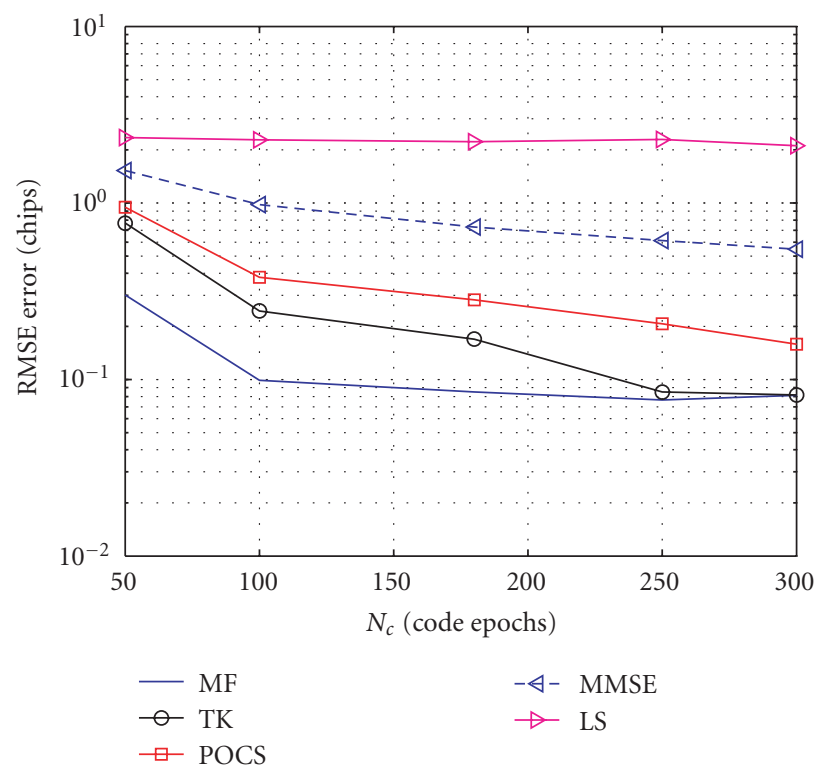

(b) Indoor channel, decaying PDP, $x_{\max }=0.1$ chips, $L_{\max }=7$ paths

FIGURE 15: Comparison of feedforward delay estimation algorithms as a function of $N_{c}$, indoor channel, decaying PDP, $x_{\max }=0.1$ chips, $N_{n c}=8, \mathrm{CNR}=22 \mathrm{~dB}-\mathrm{Hz}, N_{\mathrm{BOC}}=2, v=4 \mathrm{~km} / \mathrm{h} . P_{d}$ within $\Delta \varepsilon_{P_{d}}=0.025$ chips error (a) and RMSE in chips (b).

paths (i.e., they typically have the best detection probabilities compared to the other algorithms).

Figure 14 shows the impact of the increasing $N_{\mathrm{BOC}}$, for the indoor scenarios with maximum path spacing $x_{\max }=$ 0.05 chips (i.e., very closely spaced paths). Similar results have been obtained for spacings up to $x_{\max }=0.2$ chips. For a fair comparison between the algorithms, we assumed that the same target $\Delta \varepsilon_{P_{d}}$ is aimed (here 0.025 chips). For MMSE and LS, the $P_{d}$ performance is deteriorating when $N_{\mathrm{BOC}}$ increases (this is partially due to the errors in the noise variance $\widehat{\sigma}^{2}$ estimation). For TK and POCS, the best $P_{d}$ performance is achieved at $N_{\mathrm{BOC}}=2$, while for MF the best $P_{d}$ is achieved at $N_{\mathrm{BOC}}=3$. This behavior is mainly due to the increase in the number and amplitude of side-lobes in the ACF, when $N_{\mathrm{BOC}}$ increases, and to the computation of the threshold, which is sensitive to the height of the side-lobes. By optimizing the choice of the threshold for each modulation order, the authors believe that the performance at higher $N_{\mathrm{BOC}}$ of all the discussed algorithms can be improved. The side-lobes of the ACF act as interferers in the estimation process, and it may happen that the delay estimate goes to one of the peaks in the vicinity of the global maximum, due to the noise. In terms of RMSE error, however, the BOC modulation order does not seem to have great impact on TK, MF, and POCS estimators. TK and POCS have much better performance than MF if the spacing between paths is much less than the width of the main lobe of the ACF, namely, $1 / N_{\mathrm{BOC}}$. The performance of MF compared to the other algorithms becomes better when the main lobe of the ACF becomes narrower, as expected.

Figure 15 shows the effect of increasing the coherent integration time $N_{c}$, at a fixed CNR of $22 \mathrm{~dB}-\mathrm{Hz}$. We see that a low CNR can be compensated by increasing the integration time (here $N_{c}$ ), and that the performance of TK, POCS, LS, and MMSE algorithms becomes better with the increase of $N_{c}$. On the other hand, MF performance (and, especially, its detection probability within 0.025 chips) does not vary much with respect to $N_{c}$, which means that MF estimator is much more robust to the noise compared to the other delay estimation algorithms, but it cannot cope with the merging paths, and therefore, its detection probability remains quite low even at high $N_{c}$.

We remark that the small decrease of MF detection probability at high CNR or high $N_{c}$ (as seen in Figures 11, 12, and 15) can be explained by the fact that the simulations were carried out for 500 random realizations for each observation interval (one observation interval has a length of $N_{c} N_{n c}$ code epochs) and that the additive noise samples were, of course, different from one CNR value to the other. In order to get smoother curves, the number of random realizations should be increased, but this will also increase the simulation time.

\section{COMPLEXITY CONSIDERATIONS}

\subsection{Implementation platform}

Different architectures and implementation platforms of mobile receiver have been developed through commercial and noncommercial organizations [48, 49]. The basic trend in today's implementation is to push the design toward programmability to simplify the analog part and have more flexibility in the digital side $[50,51]$.

To compare the implementation complexity of MF, TK, and POCS algorithms (which are those with the best performance among the other analyzed feedforward techniques), we will focus on the programmable type of implementation. In mobile positioning, the main concern for the implementation platform is the low power consumption and fast 
TABLE 3: TMS320C64x and TMS320C55x parametric.

\begin{tabular}{ccc}
\hline Parametric & TMS320C64x & TMS320C55x \\
\hline Frequency $(\mathrm{GHz})$ & $0.3-1$ & $0.144-0.3$ \\
Peak MMACS & $3200-8000$ & $320-600$ \\
Active power $(\mathrm{W})$ & $0.25-1.06$ & $0.065-0.16$ \\
Pricing $(1 \mathrm{KU})$ & $32.71-296.8$ US\$ & $4.99-2.47$ US\$ \\
\hline
\end{tabular}

computation speed. For these two reasons, we choose the two fixed point digital signal processors (DSPs) from Texas Instruments; the TMS320C64x and TMS320C55x families. The C64x family is known to be the fastest DSP with up to $1 \mathrm{GHz}$ and 8000 Peak MMACS ${ }^{5}$ performance and the C55x family architecture achieves power-efficient performance with a range of 65 to $160 \mathrm{~mW}$ (see Table 3 ).

The implementation is done using the code composer studio (CCS) from TI $[52,53]$ with mixed $\mathrm{C}$ and assembly language implementation $[54,55]$.

\subsection{Implementation analysis}

The main concern of the implementation part was to compare MF, TK, and POCS algorithm in a tracking (or fine delay estimation) mode. Therefore, we assume that the correlation part is already done in the acquisition stage, which is more likely to use a hardware type of implementation due to the intensive computation needed when long code epoch is used such as, for example, 8184 chips [2] or 4092 [56] chips in Galileo signal. In tracking mode, we assume that the first arriving path will be within the search-window length of the channel $D_{\max }{ }^{6}$ which can be couple of tens of chips in indoor propagation and can reach some hundreds of chips for outdoor signal. The implementation of POCS algorithm assumes also that the matrix $\mathbf{G}_{\mathrm{BOC}}$ and the inverses of (15) and (18) are computed only once at the beginning and they are available at internal memory of the receiver. This is not an unreasonable assumption since $\mathbf{G}_{\mathrm{BOC}}$ matrix does not depend on the used codes, but only on the BOC modulation order, as seen in Section 3.2.

In Figure 16 we show the average execution time for MF, TK, and POCS algorithms for different values of the maximum delay spread of the channel and for different BOC modulation orders when we use the TMS320C64x processor. In computing these execution times, we only included the search for local maxima algorithm (which depends on the length of the correlation or cost function in samples, and, hence, on the number of samples per chip and on the $N_{\text {BOC }}$ ), the threshold computation, and the TK and POCS processing. The sampling interval was assumed here to be very small compared to the chip duration $\left(T_{s}=0.05 T_{c} / N_{\mathrm{BOC}}\right.$, or equiv-

\footnotetext{
${ }^{5}$ Million multiply-accumulates per second.

${ }^{6}$ We remark that $d_{\max }-d_{\min }$ and $D_{\max }$ stand for the same parameter, namely, the estimated maximum delay spread of the channel (or the delay search window), but the first one is expressed in samples, and the last one is expressed in chips.
}

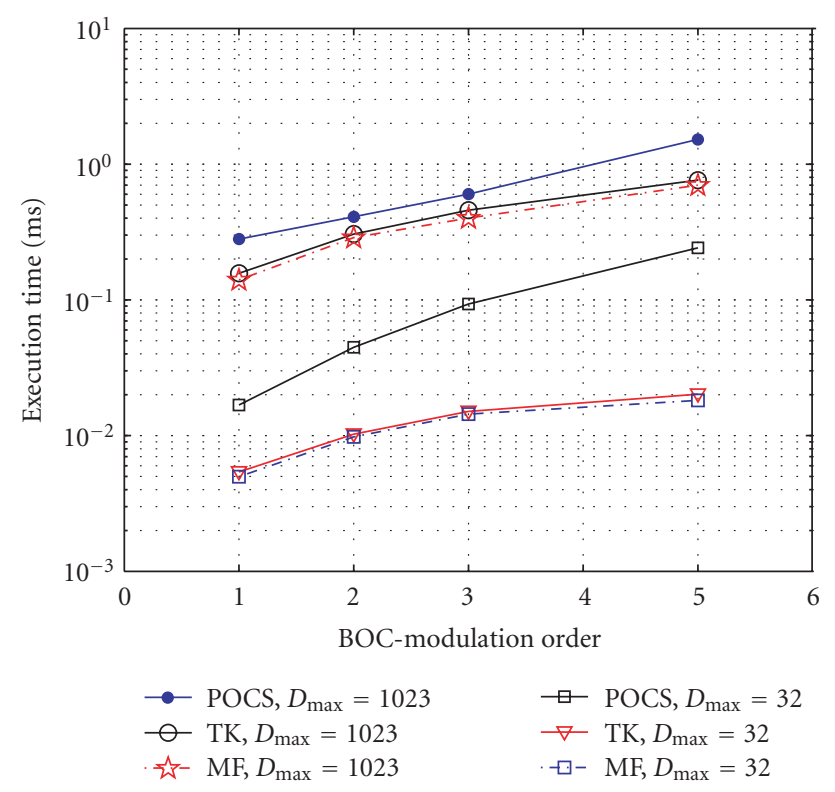

FIGURE 16: Execution time for for MF, TK, and POCS algorithms with the TMS320C64x processor at $1 \mathrm{GHz}$. Maximum delay spread $D_{\max }=32$ and 1023 chips in the presence of BOC modulation, $N_{\text {BOC }} \in\{1,2,3,5\}$.

TABle 4: Percentage of time required for delay estimation for different coherent integration times, TMS320C64x, $D_{\max }=1023$, $N_{\text {BOC }}=2$.

\begin{tabular}{ccccc}
\hline$N_{c}$ & $1 \mathrm{~ms}$ & $4 \mathrm{~ms}$ & $5 \mathrm{~ms}$ & $20 \mathrm{~ms}$ \\
\hline $\mathrm{TK}$ & $30 \%$ & $7.6 \%$ & $6.1 \%$ & $1.5 \%$ \\
POCS & $40.9 \%$ & $10.3 \%$ & $8.2 \%$ & $2 \%$ \\
$\mathrm{MF}$ & $28.8 \%$ & $7.2 \%$ & $5.7 \%$ & $1.4 \%$ \\
\hline
\end{tabular}

alently, we have $N_{s}=20$ subsamples per BOC sample, which is also the assumption used in the simulation part).

We see that, for a BOC modulation order less than 4, both in outdoor (e.g., $D_{\max }=1023$ chips) and indoor (e.g., $D_{\max }=32$ chips), the average execution time does not exceed $1 \mathrm{~ms}$. We also see that the complexity of TK is very close to the MF complexity. However, for POCS algorithm, the complexity is higher and the highest gap between TK and POCS is seen at low maximum delay spread. For $D_{\max }=1023$, TK and POCS have close computation time.

These computation times show that these algorithms can be applied quite efficiently in real-time systems. For example, in outdoor environment (case of $D_{\max }=1032$ chips), if we consider a coherent integration of $5 \mathrm{~ms}$, the percentage of time required for multipath delay estimation is less than $20 \%$ of the total time (see Table 4).

The memory requirements for MF, TK, and POCS algorithms implementation with BOC modulation order 5 are shown in Table 5 . The memory is divided into program memory (PM), data memory (DM), and external memory (Ext.M). 
TABle 5: Memory needed for TK and POCS implementation, TMS320C64x, $N_{\text {BOC }}=5$.

\begin{tabular}{cccc}
\hline$D_{\max }$ & PM (KB) & DM (KB) & Ext.M (KB) \\
\hline \multicolumn{3}{c}{ MF } \\
\hline 10 & 3.2 & 0.306 & 0 \\
32 & 16.3 & 1.2 & 0 \\
1023 & 54.2 & 2.4 & 0 \\
\hline \multicolumn{5}{c}{ TK } \\
\hline 10 & 5.6 & 1.486 & 0 \\
32 & 18.5 & 1.6 & 0 \\
1023 & 80.4 & 2.83 & 0 \\
\hline \multicolumn{5}{c}{} \\
\hline 10 & 20.2 & POCS \\
32 & 66.8 & 2.68 & 840 \\
1023 & 188.42 & 3.44 & 12154.4 \\
\hline
\end{tabular}

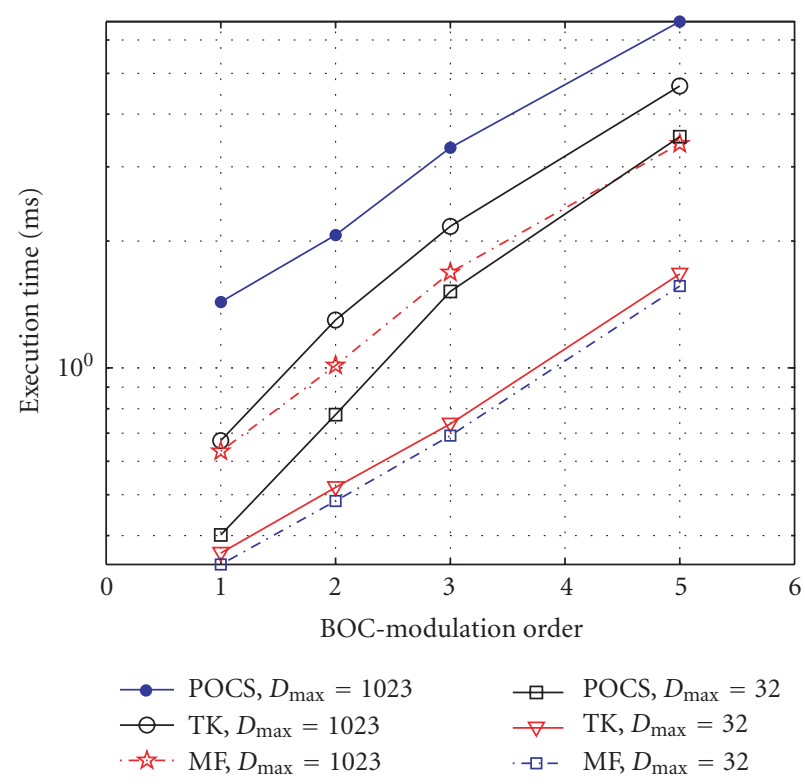

FIgURE 17: Execution time for MF, TK, and POCS algorithms with the TMS320C55x processor at $300 \mathrm{MHz}$. Maximum delay spread $D_{\max }=32$ and 1023 chips in the presence of BOC modulation, $N_{\text {BOC }} \in\{1,2,3,5\}$.

For TK algorithm, in this case for $N_{\mathrm{BOC}}=5$, the percentage of DM used does not exceed $17.68 \%{ }^{7}$ [57]. We also found that the needed memory decreases with the modulation order, as expected. For POCS algorithm, we see that the external memory is heavily used for higher maximum delay spread $D_{\max }$. This is basically due to the storage of the constant autocorrelation matrix $\mathbf{G}_{\mathrm{BOC}}$ required for POCS algorithm. For the case of $N_{\mathrm{BOC}}=5$, the percentage of DM used
TABle 6: Percentage of time required for delay estimation for different coherent integration times, TMS320C55x, $D_{\max }=1023$, $N_{\text {BOC }}=2$.

\begin{tabular}{ccccc}
\hline$N_{c}$ & $1 \mathrm{~ms}$ & $4 \mathrm{~ms}$ & $5 \mathrm{~ms}$ & $20 \mathrm{~ms}$ \\
\hline TK & $129.9 \%$ & $32.4 \%$ & $25.9 \%$ & $6.4 \%$ \\
POCS & $206.6 \%$ & $51.6 \%$ & $41.3 \%$ & $10.3 \%$ \\
MF & $101.3 \%$ & $25.3 \%$ & $20.2 \%$ & $5 \%$ \\
\hline
\end{tabular}

TABle 7: Memory needed for MF, TK, and POCS implementation, TMS320C55x, $N_{s}=20, N_{\text {BOC }}=5$.

\begin{tabular}{cccc}
\hline$D_{\max }$ & PM (KB) & DM $(\mathrm{KB})$ & Ext.M (KB) \\
\hline \multicolumn{4}{c}{ MF } \\
\hline 10 & 3.12 & 0.604 & 0 \\
32 & 5.26 & 1.92 & 0 \\
1023 & 48.8 & 4.26 & 0 \\
\hline \multicolumn{5}{c}{ TK } \\
\hline 10 & 4.2 & 1.64 & 0 \\
32 & 12.6 & 2.42 & 0 \\
1023 & 54.1 & 5.86 & 620 \\
\hline \multicolumn{5}{c}{ POCS } \\
32 & 12.8 & 3.68 & 904 \\
1023 & 42.2 & 6.44 & 12240 \\
\hline
\end{tabular}

does not exceed $31.8 \%$ and the percentage of total addressable external memory is less than $0.95 \%{ }^{8}$ [57].

The results of the implementation of TK, POCS, and MF with the TMS320C55x are shown in Figure 17. We can see that the computation time is much higher than the case of TMS320C64x. For example, in the case of TK algorithm, at $D_{\max }=1023$ and $N_{\mathrm{BOC}}=5$, the execution time with TMS320C55x is $4.67 \mathrm{~ms}$, and with TMS320C64x it is only $0.76 \mathrm{~ms}$. However, by using TMS320C55x, we expect to have lower power consumption than with the TMS320C64x.

With these execution times, the percentage of time used for multipath delay estimation with respect to the coherent integration time can be expressed in Table 6. It is clear that if we use a coherent integration of $1 \mathrm{~ms}$, the processor can not achieve the delay estimation within the required time. However, by increasing the coherent integration, the frequency of estimating the delays decreases and the operation can be achieved within the required time (e.g., with $N_{c}=5 \mathrm{~ms}$, the time allocated to delay estimation is around $41 \%$ of the total time).

The memory requirements for MF, TK, and POCS algorithms implementation with TMS320C55x are shown in Table 7 with BOC modulation order 5 . We can see that with this processor, the PM is much lower than in the case of TMS320C64x, but in overall, the total memory consumption is comparable to the case of TMS320C64x. The overall

\footnotetext{
${ }^{7}$ TMS320C64x has 16 KB data memory cache (L1D 128 K-Bit).
}

${ }^{8}$ TMS320C64x has $1280 \mathrm{MB}$ total addressable external memory space. 
memory consumption for the $D_{\max }=1023$ and $N_{\mathrm{BOC}}=5$ is about $59.9 \mathrm{~KB}$, that is, $93.5 \%$ of the total memory on-chip available $^{9}[58]$.

The external memory consumption in the case of POCS with TMS320C55x is also comparable to the case of TMS320C64x. However, the percentage of usage here is about $75.6 \%{ }^{10}[58]$.

\section{CONCLUSIONS AND DESIGN CONSIDERATIONS}

We presented here feedforward delay estimation techniques as viable alternatives for the delay tracking loops for BOCmodulated PRN signals (such as those used in Galileo and modernized GPS systems). We conclude with a discussion related to the choice of one of the feedforward techniques among those presented here. We remark that all the results regarding the detection probabilities and the RMSE values have been obtained assuming infinite bandwidth at the receiver. This allows us to obtain the bounds on the algorithm performance. Further studies are dedicated to the performance of these algorithms for bandwidth-limited receivers.

If the target in the design process is the delay estimation with very high accuracy (i.e., at most few meters), then the TK estimator is the best choice in terms of performance and complexity (it ensures the best $P_{d}$ within a very small delay estimation error and its advantage over the MF algorithm is clearly seen if the spacing between successive paths is significantly less than $1 / N_{\text {BOC }}$ chips). POCS algorithm is also better than MF algorithm in terms of separating the paths with high accuracy, but it has the drawback of a more complex implementation and requires quite many iterations (at least 10) in order to converge.

If we are rather interested to have as few outliers as possible, then MF estimator is the best choice, since it exhibits quite good RMSE curves, it has the lowest complexity, it works perfectly well when the first path is significantly stronger (in terms of average power) than the other paths, and it is the most robust to the noise level.

In order to cope with high noise levels, sufficient integration should be used. The coherent integration time is limited by the coherence time of the channel, as well as by the stability of the local oscillator at the receiver and by the residual Doppler shift errors coming from the acquisition stages. Improvements in the threshold setting may increase the performance of all the estimators (especially for TK, which is less robust to the noise than MF) and they are a topic of further investigation.

The experiments with digital signal processor implementation demonstrated that MF, TK, and POCS algorithms can be readily implemented with the current stateof-the-art, low-power DSPs, such as the TMS320C64x and TMS320C55x processors.

\footnotetext{
9 TMS320C55x has $64 \mathrm{~KB}$ on-chip RAM (32 Kx 16-bit on-chip RAM that is composed of eight blocks of $4 \mathrm{~K} \times 16$-bit dual-access RAM).

10 TMS320C55x has $8 \mathrm{M} \times 16$-bit maximum addressable external memory space, that is, $16 \mathrm{MB}$.
}

\section{ACKNOWLEDGMENTS}

This work was carried out in the project "Advanced Techniques for Mobile Positioning" funded by the National Technology Agency of Finland (Tekes). This work has also been partly supported by the Academy of Finland. The work was done when Abdelmonaem Lakhzouri was working at Tampere University of Technology.

\section{REFERENCES}

[1] G. W. Hein, J. Godet, J. L. Issler, J. C. Martin, T. Pratt, and R. Lucas, "Status of Galileo frequency and signal design," in CDROM Proceedings of the International Technical Meeting of the Institute of Navigation (ION-GPS '02), Portland, Ore, USA, September 2002.

[2] G. W. Hein, M. Irsigler, J. A. Avila Rodriguez, and T. Pany, "Performance of Galileo L1 signal candidates," in CDROM Proceedings of European Navigation Conference GNSS, Rotterdam, The Netherlands, May 2004.

[3] B. C. Barker, J. W. Betz, J. E. Clark, et al., "Overview of the GPS M code signal," in CDROM Proceedings of the ION National Meeting; Navigating into the New Millennium, Anaheim, Calif, USA, January 2000.

[4] J. W. Betz, "The offset carrier modulation for GPS modernization," in Proceedings of the National Technical Meeting of the Institute of Navigation (ION-NTM '99), pp. 639-648, San Diego, Calif, USA, January 1999.

[5] J. W. Betz and D. B. Goldstein, "Candidate designs for an additional civil signal in GPS spectral bands," Technical Papers, MITRE, Bedford, Mass, USA, January 2002.

[6] M. K. Simon, J. K. Omura, R. A. Scholtz, and B. K. Levitt, Spread Spectrum Communication Handbook, McGraw-Hill, New York, NY, USA, revised edition, 1994.

[7] R. E. Játiva and J. Vidal, "First arrival detection for positioning in mobile channels," in Proceedings of the 13th IEEE International Symposium on Personal, Indoor and Mobile Radio Communications (PIMRC '02), vol. 4, pp. 1540-1544, Lisbon, Portugal, September 2002.

[8] A. Lakhzouri, E. S. Lohan, R. Hamila, and M. Renfors, "Extended Kalman filter channel estimation for line-of-sight detection in WCDMA mobile positioning," EURASIP Journal on Applied Signal Processing, vol. 2003, no. 13, pp. 1268-1278, 2003.

[9] E. S. Lohan, Multipath delay estimators for fading channels with applications in CDMA receivers and mobile positioning, Ph.D. thesis, Tampere University of Technology, Tampere, Finland, October 2003.

[10] J. Vidal, M. Najar, and R. E. Játiva, "High resolution timeof-arrival detection for wireless positioning systems," in Proceedings of IEEE 56th Vehicular Technology Conference (VTC '02), vol. 4, pp. 2283-2287, Vancouver, BC, Canada, September 2002.

[11] N. R. Yousef and A. H. Sayed, "Detection of fading overlapping multipath components for mobile positioning systems," in Proceedings of IEEE International Conference on Communications (ICC '01), vol. 10, pp. 3102-3106, Helsinki, Finland, June 2001.

[12] D. D. Colclough and E. L. Titlebaum, "Delay-doppler POCS for specular multipath," in Proceedings of IEEE International Conference on Acoustic, Speech, and Signal Processing (ICASSP '02), vol. 4, pp. 3940-3943, Orlando, Fla, USA, May 2002. 
[13] G. Fock, J. Baltersee, P. Schulz-Rittich, and H. Meyr, "Channel tracking for rake receivers in closely spaced multipath environments," IEEE Journal on Selected Areas in Communications, vol. 19, no. 12, pp. 2420-2431, 2001.

[14] Z. Z. Kostić and G. G. Pavlović, "Resolving subchip-spaced multipath components in CDMA communication systems," IEEE Transactions on Vehicular Technology, vol. 48, no. 6, pp. 1803-1808, 1999.

[15] R. D. J. Van Nee, "The multipath estimating delay locked loop," in Proceedings of IEEE 2nd International Symposium on Spread Spectrum Techniques and Applications (ISSSTA '92), pp. 39-42, Yokohama, Japan, November-December 1992.

[16] N. R. Yousef and A. H. Sayed, "A new adaptive estimation algorithm for wireless location finding systems," in Proceedings of 33rd Asilomar Conference on Signals, Systems, and Computers, vol. 1, pp. 491-495, Pacific Grove, Calif, USA, October 1999.

[17] J. Baltersee, G. Fock, and P. Schulz-Rittich, "Adaptive codetracking receiver for direct-sequence code division multiple access (CDMA) communications over multipath fading channels and method for signal processing in a Rake receiver," US Patent Application Publication, US 2001/0014114 A1 (Lucent Technologies), August 2001.

[18] R. Bischoff, R. Häb-Umbach, W. Schulz, and G. Heinrichs, "Employment of a multipath receiver structure in a combined GALILEO/UMTS receiver," in Proceedings of 55th Vehicular Technology Conference (VTC'02), vol. 4, pp. 1844-1848, Birmingham, Ala, USA, May 2002.

[19] K.-C. Chen and L. D. Davisson, "Analysis of SCCL as a PNcode tracking loop," IEEE Transactions on Communications, vol. 42, no. 11, pp. 2942-2946, 1994.

[20] P. Fine and W. Wilson, "Tracking algorithms for GPS offset carrier signals," in Proceedings of the National Technical Meeting of the Institute of Navigation (ION-NTM '99), San Diego, Calif, USA, January 1999.

[21] M. Laxton, "Analysis and simulation of a new code tracking loop for GPS multipath mitigation,” M.S. thesis, Air Force Institute of Technology, Dayton, Ohio, USA, 1996.

[22] A. J. Van Dierendonck, P. Fenton, and T. Ford, "Theory and performance of narrow correlator spacing in a GPS receiver," Navigation, vol. 39, no. 3, pp. 265-283, 1992.

[23] M. Irsigler and B. Eissfeller, "Comparison of multipath mitigation techniques with consideration of future signal structures," in Proceedings of the International Technical Meeting of the Institute of Navigation (ION-GPS/GNSS '03), pp. 25842592, Portland, Ore, USA, September 2003.

[24] G. A. McGraw and M. Braasch, "GNSS multipath mitigation using high resolution correlator concepts," in Proceedings of the National Technical Meeting of the Institute of Navigation (ION-NTM '99), pp. 333-342, San Diego, Calif, USA, January 1999.

[25] L. Garin and J. M. Rousseau, "Enhanced strobe correlator multipath rejection for code and carrier," in Proceedings of the International Technical Meeting of the Institute of Navigation (ION-GPS '97), pp. 559-568, Kansas City, Mo, USA, September 1997.

[26] P. Fenton, B. Smith, and J. Jones, "Theory and performance of the pulse aperture correlator," Tech. Rep., NovAtel, Calgary, Alberta, Canada, September 2004. (active May 2005), http://www.novatel.com/customer_support/alltechpapers. html.

[27] L. R. Weill, "Multipath mitigation—how good can it get with new signals?" GPS World, vol. 16, no. 6, pp. 106-113, 2003.
[28] W. H. Sheen and G. L. Stüber, "A new tracking loop for direct sequence spread spectrum systems on frequency-selective fading channel," in Proceedings of IEEE International Conference on Communications (ICC '95), vol. 3, pp. 1364-1368, Seattle, Wash, USA, January 1995.

[29] R. A. Iltis, "Joint estimation of PN code delay and multipath using the extended Kalman filter," IEEE Transactions on Communications, vol. 38, no. 10, pp. 1677-1685, 1990.

[30] E. S. Lohan, R. Hamila, A. Lakhzouri, and M. Renfors, "Highly efficient techniques for mitigating the effects of multipath propagation in DS-CDMA delay estimation," IEEE Transactions on Wireless Communications, vol. 4, no. 1, pp. 149-162, 2005.

[31] J.-J. Fuchs, "Multipath time-delay detection and estimation," IEEE Transactions on Signal Processing, vol. 47, no. 1, pp. 237243, 1999.

[32] E. S. Lohan and M. Renfors, "Feedforward approach for estimating the multipath delays in CDMA systems," in Proceedings of Nordic Signal Processing Symposium (NORSIG '00), vol. 1, pp. 125-128, Kolmården, Sweden, June 2000.

[33] Z. Z. Kostić, M. I. Sezan, and E. L. Titlebaum, "Estimation of the parameters of a multipath channel using set-theoretic deconvolution," IEEE Transactions on Communications, vol. 40, no. 6, pp. 1006-1011, 1992.

[34] R. Hamila, Synchronization and multipath delay estimation algorithms for digital receivers, Ph.D. thesis, Tampere University of Technology, Tampere, Finland, June 2002.

[35] R. Hamila, E. S. Lohan, and M. Renfors, "Novel technique for multipath delay estimation in GPS receivers," in Proceedings of International Conference on Third Generation Wireless and Beyond (3GWireless '01), vol. 1, pp. 993-998, San Francisco, Calif, USA, June 2001.

[36] E. S. Lohan, A. Lakhzouri, and M. Renfors, "Spectral shaping of Galileo signals in the presence of frequency offsets and multipath channels," in Proceedings of 14th IST Mobile \& Wireless Communications Summit, Dresden, Germany, June 2005.

[37] E. S. Lohan, A. Lakhzouri, and M. Renfors, "Double BinaryOffset-Carrier (DBOC) modulation technique for satellite systems," Tech. Rep. ISBN 952-15-1348-9, ISSN 1459.4617, Institute of Communications Engineering, Tampere University of Technology, Tampere, Finland, April 2005.

[38] E. Rebeyrol, C. Macabiau, L. Lestarquit, L. Ries, and J. L. Issler, "BOC power spectrum densities," in CDROM Proceedings of the National Technical Meeting of the Institute of Navigation (ION-NTM '05), San Diego, Calif, USA, January 2005.

[39] F. Bastide, O. Julien, C. Macabiau, and B. Roturier, "Analysis of L5/E5 acquisition, tracking and data demodulation thresholds," in Proceedings of the International Technical Meeting of the Institute of Navigation (ION-GPS '02), pp. 2196-2207, Portland, Ore, USA, September 2002.

[40] T. S. Rappaport, Wireless Communications: Principles and Practice, Prentice-Hall, Englewood Cliffs, NJ, USA, 1996.

[41] D. Betaille, J. Maenpa, and P. Cross, "Overcoming the limitations of the phase multipath mitigation window," in Proceedings of the International Technical Meeting of the Institute of Navigation (ION-GPS/GNSS '03), pp. 2102-2111, Portland, Ore, USA, September 2003.

[42] R. Hamila, E. S. Lohan, and M. Renfors, "Subchip multipath delay estimation for downlink WCDMA system based on Teager-Kaiser operator," IEEE Communications Letters, vol. 7, no. 1, pp. 1-3, 2003. 
[43] E. S. Lohan and M. Renfors, "A novel deconvolution approach for high accuracy LOS estimation in WCDMA environments," in Proceedings of 7th International Symposium on Signal Processing and Its Applications (ISSPA '03), vol. 2, pp. 299-302, Paris, France, July 2003.

[44] Z. Kostic and G. Pavlovic, "Resolving sub-chip spaced multipath components in CDMA communication systems," in Proceedings of 43rd IEEE Vehicular Technology Conference (VTC '93), vol. 1, pp. 469-472, Secaucus, NJ, USA, May 1993.

[45] H. Trussell and M. Civanlar, "Feasible solution in signal restoration," IEEE Transactions on Acoustics, Speech, and Signal Processing, vol. 32, no. 2, pp. 201-212, 1984.

[46] J. F. Kaiser, "On a simple algorithm to calculate the 'energy' of a signal," in Proceedings of IEEE International Conference on Acoustics, Speech, and Signal Processing (ICASSP '90), vol. 1, pp. 381-384, Albuquerque, NM, USA, April 1990.

[47] J. Baltersee, "Modeling \& simulating fading channels for systems with smart antennas," in Proceedings of the 9th IEEE International Symposium on Personal, Indoor and Mobile Radio Communications (PIMRC '98), vol. 2, pp. 957-961, Boston, Mass, USA, September 1998.

[48] R. Baines, “The DSP bottleneck," IEEE Communication Magazine, vol. 33, no. 5, pp. 46-54, 1995.

[49] R. I. Lackey and D. W. Upmal, "Speakeasy: the military software radio," IEEE Communications Magazine, vol. 33, no. 5, pp. 56-61, 1995.

[50] E. Buracchini, "Software radio concept," IEEE Communications Magazine, vol. 38, no. 9, pp. 138-143, 2000.

[51] W. H. W. Tuttlebee, "Software radio technology: a European perspective," IEEE Communications Magazine, vol. 37, no. 2, pp. 118-123, 1999.

[52] Texas Instruments, Code Composer Studio User's Guide, March 2005.

[53] Texas Instruments, Code Composer Studio v3.0 Getting Started Guide, May 2000.

[54] Texas Instruments, TMS320C54x Optimizing C/C++ Compiler User's Guide, October 2002.

[55] Texas Instruments, TI, TMS320C6x Optimizing C Compiler: User's Guide, February 1998.

[56] Galileo Joint Undertaking (GJU), "Galileo standardization document for 3gpp," GJU webpages, (active December 2005), May 2005, http://www.galileoju.com.

[57] Texas Instruments, TMS320C64x Datasheet, March 2005, http://www.ti.com.

[58] Texas Instruments, TMS320C55x Datasheet, November 2004, http://www.ti.com.

Elena Simona Lohan received the M.S. degree in electrical engineering from the Politehnica University of Bucharest, Romania, in 1997, the D.E.A. degree in econometrics from Ecole Polytechnique, Paris, France, in 1998, and the Doctor of Technology degree in telecommunications from Tampere University of Technology, Tampere, Finland, in 2003. She is currently a Senior Researcher in the Institute of Communications Engineer-

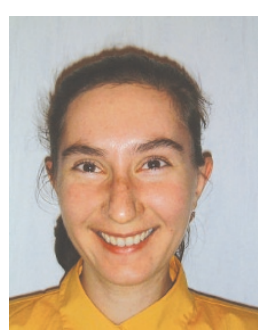
ing, Tampere University of Technology. Her research interests include GPS/Galileo positioning techniques, CDMA signal processing, and wireless channel modeling and estimation.
Abdelmonaem Lakhzouri was born in Tunis, Tunisia, on January 1, 1975. He received the M.S. degree in signal processing from the Ecole Suprieure des Communications de Tunis, Tunisia in 1999, the Diplôme d'Etudes Approfondies (DEA) degree in telecommunications from Ecole $\mathrm{Na}$ tionale d'Ingénieurs de Tunis, Tunisia in 2001, and the Doctor of Technology degree in telecommunications from Tampere Uni-

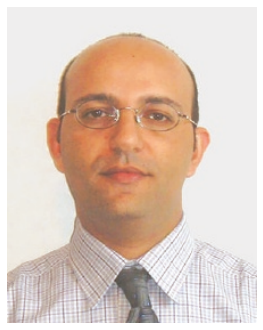
versity of Technology, Tampere, Finland, in 2005. From 2000 till March 2006 he was a Researcher at the Institute of Communication Engineering, Tampere University of Technology, Finland. Now, he is with u-Nav Microelectronics, as a Satellite Navigation Specialist.

Markku Renfors was born in Suoniemi, Finland, on January 21, 1953. He received the Diploma Engineer, Licentiate of Technology, and Doctor of Technology degrees from Tampere University of Technology (TUT), Tampere, Finland, in 1978, 1981, and 1982, respectively. From 1976 to 1988 , he held various research and teaching positions at TUT. From 1988 to 1991, he was a Design Manager at the Nokia Research Cen-

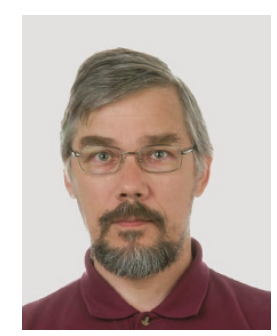
ter and Nokia Consumer Electronics, Tampere, Finland, where he focused on video signal processing. Since 1992, he has been a Professor of telecommunications at TUT. His main research area is signal processing algorithms for flexible radio receivers and transmitters. 